\title{
Strain-driven orbital and magnetic orders and phase separation in epitaxial half-doped manganite films for tunneling devices
}

\author{
D. Pesquera ${ }^{1}$, A. Barla ${ }^{2}$, M. Wojcik ${ }^{3}$, E. Jedryka ${ }^{3}$, F. Bondino ${ }^{4}$, E. Magnano ${ }^{4}$, S. Nappini ${ }^{4}$, D. Gutiérrez ${ }^{1}$, \\ G. Radaelli ${ }^{1,5}$, G. Herranz ${ }^{1}$, F. Sánchez ${ }^{1}$ and J. Fontcuberta ${ }^{1}$ \\ ${ }^{1}$ Institut de Ciència de Materials de Barcelona ICMAB-CSIC, Campus UAB, 08193 Bellaterra, Spain \\ ${ }^{2}$ Istituto di Struttura della Materia, ISM CNR, S.S. 14 km 163.5, Area Science Park Basovizza (Ts), Trieste I- \\ 34149 , Italy \\ ${ }^{3}$ Polish Academy of Science, Institute of Physics, Al. Lotnikow 32-46, PL-02668 Warsaw, Poland \\ ${ }^{4}$ Laboratorio TASC, IOM CNR, S.S. 14 km 163.5, Area Science Park Basovizza (Ts), Trieste I-34149, Italy \\ ${ }^{5}$ LNESS-Dipartimento di Fisica-Politecnico di Milano, Via Anzani 42, 22100 Como, Italy
}

\begin{abstract}
Mixed-valence manganites $\mathrm{La}_{1-\mathrm{x}} \mathrm{A}_{x} \mathrm{MnO}_{3}(\mathrm{~A}=\mathrm{Sr}, \mathrm{Ca})$ with $\mathrm{x} \approx 0.5$ can be driven from a ferromagneticmetallic to an antiferromagnetic-insulating state by a small modification $(\Delta x)$ of the carrier density $(\Delta x / x<1)$. For this reason these oxides have received a renewed attention due to their potentially advantageous integration in ferroelectric tunnel junctions of adjustable tunnel barrier width. Interestingly, in thin films, epitaxial strain can modify the electronic and magnetic ground state strongly affecting their magnetotransport properties. Here we exploit the extreme sensitivity of linearly/circularly polarized $\mathrm{x}$-ray absorption to orbital anisotropy and magnetic ordering to explore the role of structural distortions and electronic bandwidth on the orbital occupancy and spin ordering of $\mathrm{Mn}-3 d$ states in $\mathrm{La}_{0.5} \mathrm{~A}_{0.5} \mathrm{MnO}_{3}$ films under various strain states. ${ }^{55} \mathrm{Mn}$ NMR experiments are used to get information about the electronic and magnetic phase separation and orbital ordering occurring in these films. These results, combined with the corresponding structural, magnetic and electrical characterization, allow to map the strain-dependent orbital and magnetic phase diagrams of half-doped manganites and its dependence on the electronic bandwidth.
\end{abstract}

\section{INTRODUCTION}

Manganites have been considered as one of the most promising materials for achieving an effective electrical manipulation of magnetic properties, leading to the creation of magnetoelectric devices to replace current memory technologies [1]. In ferroelectric/manganite heterostructures, modifications of the charge carrier concentration, strain or chemical bonds can occur at the interface and this modification can stabilize different ground states in the manganite layer $[2,3]$. These effects are expected to be exceptionally effective at manganite compositions close to the boundary between two magnetic phases. That is the case of half-doped (HD) manganites of composition $\mathrm{La}_{1-x} \mathrm{~A}_{x} \mathrm{MnO}_{3}(\mathrm{~A}$ $=\mathrm{Ca}$ and $\mathrm{Sr}$ ) with $\mathrm{x} \approx 0.5$. The bulk phase diagrams of these materials [4] show that they are at the verge between ferromagnetic (FM) metallic $(x<0.5)$ and antiferromagnetic (AF) insulating $(x>0.5)$ phases. Integration of ultrathin layers of these materials adjacent to a ferroelectric layer in ferroelectric tunnel junctions, could in principle allow switching on/off their metallic/insulating nature upon the reversal of the ferroelectric polarization by promoting a charge injection/depletion, i.e. by polarization-controlled field effect. By inducing a metal to insulator transition in the manganite, a modification in the tunnel barrier width could lead to a giant tunnel electroresistance [5], much larger than in conventional $\mathrm{BaTiO}_{3}$-based ferroelectric tunnel junctions $[5,6,7]$ and even larger than in $\mathrm{BaTiO}_{3}$ ferroelectric barriers with polarization dependent depletion layer in the semiconducting ferroelectric layer [8]. However, although such heterostructures have been fabricated yielding promising results [9], the actual properties and response of the ultrathin HD layer required for tunnel devices may differ from the known properties of nanometric layers [10].

A first step to understand and optimize the materials to be intercalated in the ferroelectric junction is to consider the strain effects on the HD manganite layers, whose electronic properties are known to be extremely sensitive to charge or orbital orderings [11, 12]. In fact, the strain-dependent phase diagram in these compounds has been explored by first-principles band-structure calculations [13], demonstrating that in tetragonally-distorted films with a doping $0.2<x<0.6$ the ground state can be 
tuned from C-type antiferromagnetic insulating (C-AF/I) to ferromagnetic metallic (FM/M) and to $A-$ type antiferromagnetic metallic (A-AF/M) by changing the strain from compressive (elongated c-axis) to tensile (compressed c-axis). This magnetic and electronic phase modulation had already been experimentally demonstrated in $\mathrm{La}_{1-x} \mathrm{Sr}_{x} \mathrm{MnO}_{3}$ films grown on different substrates by Konishi et al. [14]. However, the strain is not the only relevant factor, since the electronic bandwidth seems to play a key role. For instance, Gutiérrez et al. [10] recently showed, by means of an exhaustive study of the transport and magnetic properties of $\mathrm{La}_{0.5} \mathrm{Sr}_{0.5} \mathrm{MnO}_{3}$ (LSMO) and $\mathrm{La}_{0.5} \mathrm{Ca}_{0.5} \mathrm{MnO}_{3}$ (LCMO) thin films, that the magnetization and metallic behaviour of LSMO films can be largely controlled by strain, while LCMO films remain in a non-FM insulating state independently of the applied strain. The narrower bandwidth of LCMO seems to play a relevant role, by promoting electron localization and favouring the appearance of electronic phase separation and charge localization as already observed in bulk HD manganites [15].

On the basis of the well-established influence of surface and interfaces on the electronic states we anticipate that both strain and bandwidth can have a nontrivial interplay in the determination of the orbital character of electrons participating in the tunnel transport. Bearing this in mind, here we used element sensitive $\mathrm{x}$-ray absorption measurements to probe the orbital occupancy of the $\mathrm{Mn}$ $3 d\left(e_{g}\right)$ levels, thus disclosing the effects of strain on the breaking of the degeneracy of the $3 d\left(e_{g}\right)$ manifold. In the same experiment, at temperatures below the magnetic ordering of the films, we examined the presence (and magnetic orientation) of FM and/or AF phases. In this way, a direct connection between orbital and spin degrees of freedom is established. On the other hand, we use ${ }^{55} \mathrm{Mn}$ NMR to determine the hyperfine magnetic field acting on ${ }^{55} \mathrm{Mn}$ nuclei which is known to be an extremely sensitive probe of nanoscale electronic and magnetic properties $[16,17,18,19]$.

Our measurements show that in LSMO, strain controls the occupancy of either $x^{2}-y^{2}$ or $3 z^{2}-r^{2}$ orbitals and resulting from this orbital selection, different magnetic ground states are stabilized, as expected from calculations [13], while in LCMO a more complex scenario involving different orbital orderings drives the system to a mostly AF state. NMR data provides microscopic evidence of strain induced electronic phase separation and of magnetic hardening under compressive stress. In our study, tensile and compressive strain states are explored and the surface contribution to the orbital occupancy is disentangled from the purely elastic response thus largely expanding recent works [20] where only tensile effects on properties of HD LSMO films were addressed.

The results presented here provide a comprehensive spectroscopic view of strain effects on halfdoped manganites with particular focus on orbital occupancy and magnetic order and the distinct response of $\mathrm{La}_{1 / 2}(\mathrm{Ca}, \mathrm{Sr})_{1 / 2} \mathrm{MnO}_{3}$ to strain depending on bandwidth. The results described here should help to better understand the response of ultrathin HD manganite films which could be of relevance for the quest towards more responsive ferroelectric tunnel barriers.

\section{EXPERIMENT}

$\mathrm{La}_{0.5} \mathrm{Sr}_{0.5} \mathrm{MnO}_{3}$ and $\mathrm{La}_{0.5} \mathrm{Ca}_{0.5} \mathrm{MnO}_{3}$ films were epitaxially grown by pulsed laser deposition on (001)oriented single-crystalline substrates with cubic or nearly cubic crystal structure: $\mathrm{DyScO}_{3}$ (DSO, lattice constant of $3.940 \AA$ ) , $\mathrm{SrTiO}_{3}$ (STO, $3.905 \AA$ ) ), (La,Sr)(Al,Ta)O ${ }_{3}$ (LSAT, $3.870 \AA$ ), $\mathrm{LaAlO}_{3}(\mathrm{LAO}$, $3.792 \AA$ ) and $\mathrm{YAIO}_{3}$ (YAO, $3.720 \AA$ ). Pseudocubic cell dimensions are given for orthorhombic substrates. The deposition conditions are specified in ref.[10]. All films were grown with a thickness of $20 \mathrm{~nm}$, as checked by $\mathrm{x}$-ray reflectivity. The calculated mismatch values between the pseudocubic lattice parameter of LSMO (3.858 $)$ or LCMO $(3.830 \AA)$ and the crystalline substrates are shown in Table 1, together with the ratio between out-of-plane (c) (ref.[10]) and in-plane lattice parameters (a). The mismatch between films and substrates produces a biaxial strain that tunes the tetragonality of the films, leading to a broad range of $c / a$ values that allows an extensive study of strain effects (both compressive and tensile) on half-doped manganite films. Structural, transport and magnetic characterization of the films was reported in ref.[10]. 
X-ray absorption experiments were performed at the BACH beamline of the Elettra synchrotron radiation source. Spectra were taken in total electron yield mode (TEY) at energies corresponding to the $M n-L_{2,3}$ edges ( $2 p-3 d$ dipolar transitions), in grazing incidence configuration ( $x$-rays impinging at $30^{\circ}$ from the sample surface). While orbital order was probed at $\mathrm{T}=300 \mathrm{~K}$, above any magnetic ordering of the films, magnetic orders were probed at low temperatures ( $T=5 \mathrm{~K}$ for $L S M O$ and $T=50$ $\mathrm{K}$ for LCMO [21]), in the presence of a magnetic field parallel to the $\mathrm{x}$-rays propagation direction, large enough to saturate the magnetic moments $(B=2 T)$, according to the macroscopic measurements of the samples having a measurable magnetic signal [10].

Linearly polarized spectra were taken with photon polarizations either parallel $\left(E_{a b}\right)$ or perpendicular $\left(E_{c}\right)$ to the surface of the samples, and the linear dichroism (XLD) was calculated as the difference in absorption $\mathrm{I}_{a b}-I_{c}$, where the misalignment from out-of-plane direction of $E_{c}$ was taken into account by applying a geometrical correction as specified in [22]. $X$-ray absorption spectra $\left(X A S=\left(I_{a b}+I_{c}\right) / 2\right)$ were normalized to the intensity at the photon energy $\mathrm{E}=642 \mathrm{eV}$ and, prior to the extraction of dichroic spectra, the intensities of individual spectra were matched at energies below the $\mathrm{Mn}-\mathrm{L}_{3}$ edge, resulting in perfect intensity matching at energies above the $M n-L_{2}$ edge as well. Circularly polarized spectra were taken with right $\left(\mathrm{E}_{\text {right }}\right)$ and left $\left(\mathrm{E}_{\text {left }}\right)$ circularly polarized photons. The magnetic circular dichroism (XMCD) spectra were obtained as the difference $I_{\text {right }}{ }^{-}{ }_{\text {left }}$, after performing the same normalization procedure used for linearly polarized spectra.

NMR experiments were carried out at $4.2 \mathrm{~K}$ with the use of a coherent broadband spin echo spectrometer. The spectra were recorded with the driving field $h_{1}$ applied in-plane and with no applied DC magnetic field $(H=0)$. The experimental data were corrected for the distribution of the NMR enhancement factor $\eta$ and for its frequency variation using a protocol elaborated by $P$. Panissod and described in [23]. The corrected NMR spectrum computed in such way represents the intensity distribution which is proportional only to the number of nuclei resonating at a given NMR frequency $\mathrm{f}$. Application of the Panissod protocol makes it also possible to determine the mean value of the enhancement factor $\eta$ by measuring $h_{1 \text { opt }}(f)$, i.e. the r.f. field amplitude that maximises the spin echo NMR signal. In addition, knowing $h_{10 p t}$ one can calculate the (frequency dependent) NMR restoring field $\mathrm{H}_{\text {rest }}$ which is defined as $\mathrm{H}_{\text {rest }}=(\mathrm{HF} / \eta)=\beta \mathrm{h}_{\text {1opt }}$ where $\mathrm{HF}$ is the internal magnetic field sensed by the nucleus, and $\beta$ is the instrumental scaling factor [23]. The restoring field $H_{\text {rest }}$ is the internal magnetic field acting on the local electronic magnetization and provides an insight into the material local magnetic stiffness in different parts of the sample. The experiments were performed on a sample of LSMO or LCMO film with the lateral dimensions of $5 \times 5 \mathrm{~mm}$, which was placed in a tight fitting flat coil to maximize the filling factor. Bulk sample containing several tenth of $\mathrm{mg}$ of LSMO powder glued on a tape with the dimension $5 \times 5 \mathrm{~mm}$ was measured using the same coil in order to make the $\beta$ factor very similar to the one in case of the thin film sample and to facilitate the comparison between the respective data.

\section{RESULTS AND DISCUSSION}

\section{A. X-ray absorption}

The XAS spectra obtained with linearly polarized radiation are shown in Figures $\mathbf{1}(\mathbf{a})$ and $\mathbf{1}$ (b) for LSMO and LCMO samples, respectively. While all LCMO samples and the LSMO sample on the DSO substrate, which imposes the largest tensile strain, show an almost featureless $M n-L_{23} X A S$ characteristic of the expected $\mathrm{Mn}^{3+} / \mathrm{Mn}^{4+}$ contributions, most of the LSMO samples show a double peak structure in the lower energy region of the $L_{3}$ edge, at $\approx 639.5-640.5 \mathrm{eV}$. The difference between a XAS spectrum presenting this double peak structure and the one not showing it results in a spectrum that can be well reproduced by a simulated $\mathrm{Mn}$ spectrum with single $2+$ valence (spectrum labelled "Mn2+ (sim)" at the bottom of Figure 1(a) [24,25]). As an example we report the difference between the spectrum on LSAT and on DSO (cyan curve labelled "LSAT-DSO" in Figure 1(a)). The observation of reduced $\mathrm{Mn}^{2+}$ species in XAS experiments on manganites is rather 
common; its origin has been attributed to the existence of atmospheric reducing agents chemically interacting with the $\mathrm{La}(\mathrm{Ca}, \mathrm{Sr}) \mathrm{MnO}_{3}$ surface $[26,27]$ or to beam-stimulated oxygen desorption [28]. The prominence of this $\mathrm{Mn}$ double peak structure is observed to vary with the strain of the films. A similar strain effect had also been observed earlier in $\mathrm{RNiO}_{3}$ films [see for instance the supplementary information in Ref.29]. This could be explained as the result of a surface relaxation by promoting the formation of oxygen vacancies, thus favouring the appearance of surface $\mathrm{Mn}^{2+}$ states. The film with the largest tensile strain (LSMO on DSO), although showing a coherent epitaxial growth, has extended fracture microcracks [10] that may allow to alleviate the elastic strain energy; consistently, the $\mathrm{Mn}^{2+}$ feature is absent in the XAS of this sample. Nevertheless, the reason for the larger contents of $\mathrm{Mn}^{2+}$ on LSMO samples with respect to LCMO (Figure 1(b)) cannot be clarified by the data currently at hand. In the Supplementary Material S1 [30] we study the influence of $\mathrm{Mn}^{2+}$ on the orbital and magnetic response of the films and explore possible pathways to diminish its contribution.

\section{B. Orbital order}

Figure 1(c) shows the linear dichroic spectra (XLD) of LSMO samples, calculated as $\mathrm{I}_{a b}{ }^{-} \mathrm{I}_{\mathrm{c}}$. A clear evolution of the XLD signal with the tetragonal distortion of the films is evidenced in Figure 1(c), most clearly visible at the $L_{2}$ edge (i.e., above $648 \mathrm{eV}$ ), where samples under compressive strain (LAO, YAO, $c / a>1$ ) show a positive XLD signal, corresponding to $I_{a b}>I_{c}$, which implies a larger number of accessible states for in-plane photon polarization or, equivalently, a larger occupancy of out-ofplane oriented states $\left(3 z^{2}-r^{2}\right)$. The opposite is found for tensile strained films (DSO, STO, c/a<1) where a negative XLD signal is observed, indicating larger occupancy of planar orbitals $\left(x^{2}-y^{2}\right)$. The XLD spectral shapes resemble those reported previously for half-doped manganite LSMO films grown both on LAO and STO substrates [31]. The film grown on LSAT, although having a nearly cubic structure $(c / a \approx 0.99)$, has a clearly positive XLD. This positive dichroism, indicative of a preferential occupancy of $3 z^{2}-r^{2}$ states, can be attributed to the surface symmetry breaking, favouring the stabilization of out-of-plane oriented orbitals at the surface layers [32, 33, 34].

The relative hole population of $3 \mathrm{~d}\left(\mathrm{e}_{\mathrm{g}}\right)$ states is, according to the XLD sum rules [35], proportional to the area under the XLD signal, $I_{X L D} \propto h_{x^{2}-y^{2}}-h_{3 z^{2}-r^{2}},\left(h_{x^{2}-y^{2}}\right.$ and $h_{3 z^{2}-r^{2}}$ are the hole occupancies of $x^{2}-y^{2}$ and $3 z^{2}-r^{2}$ states). In order to monitor the dependence of the orbital occupancy on the strain we integrated the XLD signal at the $M n-L_{2}$ edge $(648-662 \mathrm{eV}$, shaded regions in Fig. $1(\mathrm{c})$ ), as in this energy range the signal is not strongly affected by the presence of the $\mathrm{Mn}^{2+}$ peak nevertheless, extending the integration range to include $L_{3}$ edge yields similar results (see Supplementary Material S2 [30])

In Figure 2 we include the obtained values for $I_{x L D}$ as a function of strain. We can highlight two aspects in this plot: a) IXLD shows a nearly linear dependence on the tetragonality of the films; this observation is in agreement with the expected degeneracy removal by means of Jahn-Teller-like distortion of the Mn-oxygen coordination polyhedron under substrate-induced strain, b) an asymmetry in the plot, displacing the values towards positive $I_{X L D}$ (larger $3 z^{2}-r^{2}$ electron occupancy), as expected from the contribution of symmetry breaking at the surface layers explained above. Therefore in HD LSMO the orbital occupancy can be well described by the joint contribution of the substrate-induced tetragonal distortion of the LSMO unit cell (represented by the tetragonality ratio c/a) and the additional specific surface-dependent term favouring $3 z^{2}-r^{2}$ occupancy.

The integrated area under the XLD signal provides a qualitative picture of the strain dependence of orbital occupancy. The $I_{x L D}$ values could be used to calculate the relative occupation of $3 \mathrm{~d}\left(\mathrm{e}_{\mathrm{g}}\right)$ states. Most commonly this is expressed through the orbital polarization $\mathrm{P}_{\mathrm{eg}}$ of states with $\mathrm{e}_{\mathrm{g}}$ symmetry defined as the relative difference of electron occupancies for $x^{2}-y^{2}\left(n_{x^{2}-y^{2}}\right)$ and $3 z^{2}-r^{2}\left(n_{3 z^{2}-r^{2}}\right)$ states [36,37]: $P_{e_{g}}=\left(n_{x^{2}-y^{2}}-n_{3 z^{2}-r^{2}}\right) / n_{e g}$. Naturally, an accurate definition of the total number of electrons in $e_{g}$ states $\left(n_{\mathrm{eg}}\right)$ is required in order to evaluate the orbital polarization $\mathrm{P}_{\mathrm{eg}}$. The most simple assumption that $n_{\text {eg }}$ is given by the doping level $(x=1 / 2)$, ignores the effect of metal-oxygen 
hybridization [37] and neglects the possible contribution of spurious surface $\mathrm{Mn}^{2+}$, which will lead to unreliable $\mathrm{P}_{\mathrm{eg}}$ values. Notice that the presence of $\mathrm{Mn}^{2+}$ would not modify the shape of the XLD signal (see Supplementary Material S1 [30]) but will affect the numerical evaluation of $P_{\mathrm{eg}}$ due to the added number of electrons at the $3 \mathrm{~d}$ manifold. Therefore any numerical estimate of $\mathrm{P}_{\mathrm{eg}}$ is plagued by a number of uncertainties that would render it inaccurate; consequently, in the following we will not discuss the numerical estimates of $P_{\text {eg }}$.

The XLD spectra obtained for LCMO samples are shown in Figure 1(d). A visual inspection of the $L_{2}$ edge in Fig. 1 (d) shows that the dichroic signal is similar to that of LSMO samples and roughly follows the same trend: compressively strained samples (LAO and YAO) show a positive signal $\left(\mathrm{I}_{\mathrm{XLD}}>0\right)$ whereas those under tensile strain (DSO and STO) display a negative XLD $\left(I_{\times L D}<0\right)$. Remarkably, the LCMO film grown on LSAT has a XLD > 0 . As argued in the case of LSMO//LSAT above, the free surface of the film always gives a positive contribution that may override an eventual negative compressive-strain induced orbital polarization [33]. This effect accounts for the observed $I_{x L D}>0$ signal of the LCMO film on LSAT.

Analogous to LSMO, the integrated signal IxLD of the LCMO samples (Figure 2) shows a roughly linear dependency with tetragonal distortion. However the slope is smaller than in LSMO indicating that orbital occupancy in LCMO is less sensitive to strain variations than in LSMO. This could be related to the presence of mechanisms for strain accommodation other than the Jahn-Teller (JT) like associated with the tetragonal distortion of the lattices. Octahedral rotations leading to unit cell symmetry lowering, more likely to occur in LCMO than in LSMO due to the smaller size of $\mathrm{Ca}^{2+}$, could be a possible candidate. By using in-house X-ray diffraction experiments [10] we were not capable of resolving a lower-than-tetragonal symmetry in our films. On the other hand, the reduction of the $A$ cation size (going from $\mathrm{Sr}$ to $\mathrm{Ca}$ ) also produces a bandwidth narrowing promoting electron localization, which can also lead to the stabilization of special orbital orderings. Theoretical calculations [12] show that tensile strain can stabilize an in-plane $3 x^{2}-r^{2} / 3 y^{2}-r^{2}$ zig-zag orbital order that would yield a strongly negative XLD signal [38]. This is indeed observed in our data for the LCMO film on STO, which deviates from the linear tendency followed by all other LCMO samples, yielding a negative signal much larger in magnitude than expected from the anticipated JT tendency. Further work is required to elucidate the quantitative differences observed for the strain dependence of XLD in LSMO and LCMO.

Finally, it is worth mentioning that in some cases, the orbital ordering is not dictated by the local JT splitting but other effects, most notably the gain in electronic kinetic energy via electron delocalization may override the JT-like effect as demonstrated in layered oxides (i.e. $\operatorname{La}_{0.5} \mathrm{Sr}_{1.5} \mathrm{MnO}_{4}$ ) [39]. It has been also claimed that this mechanism may also play a role in manganese perovskites either close to half-filling [40] or A-site $\mathrm{Mn}$ doped [41]. The systematic and consistent variation of orbital filling with strain shown here, seems to indicate that these alternative mechanisms do not play a major role.

\section{Magnetic order}

The observed effects of strain on the orbital order shall impact the magnetic interactions in the films. To explore these effects, we measured at low temperatures both the circular (XMCD) and linear (XMLD) magnetic dichroism at the $\mathrm{Mn} \mathrm{L}_{2,3}$-edges in order to infer the presence of ferromagnetic $(F)$ and antiferromagnetic (AF) phases, respectively.

The XMCD signal obtained at $\mathrm{T}=5 \mathrm{~K}$ under a field of $2 \mathrm{~T}$ which saturates the sample, is plotted in Figure 3(a) for the LSMO samples. A large difference in the dichroism magnitude for the different samples is observed: films with the lowest strain values (LSAT, YAO) show the largest XMCD signal, which gets reduced for increasing compressive (LAO) or tensile (STO, DSO) strain. Indeed, for the largest tensile strain (DSO) the signal vanishes, indicating the complete suppression of ferromagnetic order in this sample. The application of the XMCD sum rules [42] is here hampered by the uncertainty in the number of holes in the $3 \mathrm{~d}$ manifold and the presence of a fraction of $\mathrm{Mn}^{2+}$ ions at 
the surface of some of our samples. As indicated in the Supplementary Material S1 [30], the comparison of similar samples with different $\mathrm{Mn}^{2+}$ content allow us to disentangle a $\mathrm{Mn}^{2+} \mathrm{XMCD}$ spectrum that, after applying a rescaling factor can be subtracted to the original XMCD spectrum of each sample. The resultant spectra, indicated by the shadowed area in Figure 3(a,d) were used for the calculation of magnetic moments applying sum rules, that provide the orbital and spin magnetic moment per $\mathrm{Mn}$ atom in the explored sample depth (3-5 nm). While the orbital contribution is approximately null in all samples, the spin magnetic moment per number of holes -plotted in Figure $3(c, f)$ shows a clear evolution as a function of the tetragonality of the films.

Data in Figure 3(c) indicates that the ferromagnetic fraction of LSMO is maximum for $\mathrm{c} / \mathrm{a} \approx 1$ but when $\mathrm{c} / \mathrm{a}$ deviates from $\approx 1$, the fraction of ferromagnetic phase in the film decreases. The observed suppression of ferromagnetism by strain in HD LSMO films is in agreement with earlier macroscopic magnetic measurements [10] and indicates that the behaviour observed here is not restricted to the most superficial layers explored by XMCD measurements, but it reflects the magnetic character of the whole film. It thus follows that most likely an AF phase coexists with the F one when c/a strongly deviates from 1.

The presence of antiferromagnetic phases at low temperatures can be probed by means of linear dichroism measurements. While in the paramagnetic phase (above the Curie $T_{C}$ or Néel $T_{N}$ temperatures) XLD explores the orbital anisotropy, at low temperatures, when magnetic ordering comes into play, XLD is also sensitive to the charge-deformation produced by the spin-orbit coupling in the core and valence band levels and thus XLD can be used to examine the anisotropy of the spin axis [43]. Briefly, if the spin axis is parallel to the beam direction, there will be no difference due to the magnetic moments in the $x$-ray absorption for linear vertical and horizontal polarizations, both of which have electric field components perpendicular to the beam, and therefore the $x$-ray magnetic linear dichroism (XMLD) will be null. On the other hand, if the spin axis is oriented perpendicular to the beam direction, almost parallel to the electric field vector of one polarization and perpendicular to the other, the XMLD signal will be maximum. Furthermore, the shape of the XMLD signal will provide information on the orientation of the spin axis, relative to the beam direction.

In order to separate the ferromagnetic and antiferromagnetic contributions, a magnetic field large enough to saturate the ferromagnetic component can be applied in the direction of the beam. In that way, the ferromagnetically coupled spins, parallel to the applied field, will always be perpendicular to the photon polarization, thus cancelling their contribution to the XLD. Antiferromagnetic phases present in the sample will not be significantly affected by the magnetic field and can contribute to the measured linear dichroism. The orbital part, which had been measured at $T>T_{C}\left(T_{N}\right)$ and described above, can be subtracted from the measured XLD allowing one to obtain the XMLD and disentangle the AF contribution. The subtraction of the paramagnetic XLD is accurate enough under the assumption that it is temperature independent (i.e., for an isotropic thermal expansion of the lattice and in the absence of structural phase transitions).

We show in Figure $\mathbf{3}(\mathbf{b})$ the resultant XMLD recorded at grazing incidence, at the $\mathrm{Mn}-\mathrm{L}_{2,3}$ absorption edges, obtained from the XLD measured in LSMO samples at $5 \mathrm{~K}$ in the presence of a magnetic field $(B=2 T)$, after the subtraction of the XLD measured at $T=300 \mathrm{~K}$ (shown previously in Figure $\mathbf{1}(\mathrm{c})$ ). We first notice that, in the sample with the largest ferromagnetic contribution (LSAT), this subtraction results in a dichroism with nearly zero intensity for all energies, indicating the absence of AF phases in this sample. However, the XMLD signal is large in all remaining samples. One should remark the very different behaviour (most obvious at the $\mathrm{L}_{2}$-edge) between the XMLD of the compressively strained LSMO films (samples on LAO and YAO) and that of the tensile strained samples (STO and DSO): The XMLD spectra have inverted sign (negative-positive or positive-negative XMLD at the $\mathrm{L}_{2}$ edge) indicating reversed directions of the spin axis. For LAO and YAO films, the XMLD shape corresponds to an out-of-plane spin axis orientation, as it was shown in refs. [44, 45]. In order to confirm the c-axis orientation of the antiferromagnetically coupled magnetic moments in these 
layers, we performed XMLD measurements at $5 \mathrm{~K}$ in normal incidence. These measurements (shown in Supplementary Material S3 [30]) yield a zero dichroism, as expected from the perpendicular orientation of the spin axis and the x-ray polarization vectors, giving the same absorption for both light polarizations.

The opposite sign of the XMLD of the samples under tensile strain (STO, DSO) (bottom of Figure $\mathbf{3}(\mathbf{b})$ ) is in agreement with an in-plane AF alignment of the magnetic moments in these samples. Indeed, the spectral shape coincides with previously reported XMLD in materials showing antiferromagnetic phases with in-plane spin alignment [46].

In order to monitor the evolution of the AF spin axis direction as a function of strain, from the data in Figure $\mathbf{3}(\mathbf{b})$ we evaluated the difference of XMLD magnitude ( $\triangle \mathrm{XMLD}$ ) between the first peak observed (labelled " $\mathrm{i}_{\mathrm{p}}$ ") at the $\mathrm{Mn}-\mathrm{L}_{2}$ edge (positive for in-plane alignment) and the second peak (labelled " $\mathrm{O}_{p}$ " and positive for out-of-plane alignment). The data (open symbols in Figure 3(c)), combined with the corresponding XMCDs, indicate a wide region of phase coexistence with a predominance of AF-phase for $c / a<0.98$ to ferromagnetic phase for $0.98<c / a<1.00$ and finally to out-of-plane AF phase for $c / a>1.00$. Nevertheless, the considerable magnetic moment measured with XMCD for samples having $\mathrm{c} / \mathrm{a}>0.97$ clearly indicates a F/AF phase coexistence in these samples.

XMCD and XMLD measurements were also recorded on LCMO samples at $50 \mathrm{~K}$ [21]. The spectra of the measured samples are shown in Figures $\mathbf{3 ( d , e )}$ (the strong insulating character of the samples prevented us from obtaining a reliable dichroic signal in the samples not shown in Figure 3(d,e). The XMCD signal registered for samples under tensile strain is nearly zero, while an increasing XMCD is observed for increasing compressive strain. The calculated spin magnetic moment per number of holes is displayed in Figure 3(f) (as in the case of LSMO samples, orbital contribution was found to be irrelevant in LCMO samples). We can conclude from these measurements that HD LCMO films are largely $\mathrm{AF}$, although some residual fraction of $\mathrm{FM}$ phase coexists. This is in agreement with magnetization measurements on the same films [10]. The data indicates that a larger compressive strain would be required to stabilize a FM phase in LCMO films.

On the other hand, XMLD measurements (Figure 3(e)) show signatures of AF phases in both compressive (LAO, YAO) and tensile (STO, DSO) strained samples. As in the case of LSMO, strain controls the spin axis, stabilizing an AF alignment with out-of-plane (in-plane) spin axis for compressive (tensile) strain. The difference in intensity between the corresponding $i_{p}$ and $o_{p}$ peaks in the XMLD at the $L_{2}$ edge is plotted in Figure $\mathbf{3}(\mathbf{f})$ as a function of $c / a$, showing a similar trend as that observed for LSMO films (Figure 3(c)), although the dichroic values are smaller. This is likely due to the fact that measurements in LCMO films have been performed at higher temperature $(50 \mathrm{~K}$ instead of $5 \mathrm{~K}$ for LSMO).

\section{Orbital ordering studied by NMR}

In ferromagnetic materials the excitation and detection of the NMR signal is determined by the local magnetic susceptibility, which may have multiple origins ranging from domain wall pinning to magnetocrystalline anisotropy. Regardless of the physical mechanism behind the local NMR enhancement factor $\eta$, the applied protocol (see II.Experiment) makes it possible to determine phenomenologically the optimum driving field $h_{1}$ at each frequency and to correct the observed NMR signal amplitude for the intrinsic enhancement factor, as described in [23]. To illustrate this procedure, in Figure 4 we plot the NMR signal amplitude as a function of the driving field $h_{1}$, for a given frequency, for the series of three samples of LSMO with identical thickness but having different lattice strain. The experimental points for each sample have been fitted with a log-normal function showing clearly that the distributed character of $\eta$ leads to the situation that the NMR 
amplitude follows the theoretically predicted Gaussian distribution as a function of $\log \left(h_{1}\right)$. In addition we note that the width of the distribution is similar in the three studied samples, whereas the position of its maximum, corresponding to the $h_{10 p t}$, is strongly dependent on the lattice distortion due to strain.

The same procedure has been applied in the full frequency range, with the step of $1 \mathrm{MHz}$, in order to obtain the spectra for each thin film sample investigated in this work. At each frequency value, three parameters have been determined: (i) maximum spin echo intensity $I_{\text {opt }}$ (ii) the corresponding $h_{1 \text { opt }}$ and (iii) width of the intensity distribution sigma $(\sigma)$.

The NMR intensities $I_{0}(f)$ (hereinafter referred to as a spectrum) discussed further in this paper have been obtained as a product $I_{0}(f)=I_{\text {opt }}(f) * h_{1 \text { opt }}(f) * \sigma(f)$, thus correcting for the mean enhancement factor $\eta(f)=H F /\left(\beta^{*} h_{1 o p t}(f)\right)$ and frequency variation of sigma. In addition, the standard $1 / f^{2}$ factor has been applied.

Figure 5 presents ${ }^{55} \mathrm{Mn}$ NMR spectra at $4.2 \mathrm{~K}$, elaborated with the use of above procedure, for the LSMO films $(20 \mathrm{~nm})$ grown on STO, LSAT and YAO substrates, respectively, thus representing different stress state. For comparison, in the panel presenting the unstrained sample grown on LSAT, we also show the NMR spectrum recorded from a bulk (ceramic sample) of half-doped LSMO. In case of the highly strained LSMO films, grown on LAO and DSO substrates, ${ }^{55} \mathrm{Mn}$ NMR signal could not be observed in the experimental conditions suitable for detecting NMR signal from a ferromagnetic material. Likewise, the ${ }^{55} \mathrm{Mn}$ NMR signal was not observed in any of the studied LCMO films, independently of the substrate and corresponding strain. It will be shown that this is consistent with the XMCD data reported above. The same situation was observed in case of $36 \mathrm{~nm}$ thick films: the signal could only be detected in LSMO films, and only in case of the three substrates mentioned above. The NMR spectra recorded from $36 \mathrm{~nm}$ films were almost identical to those of $20 \mathrm{~nm}$, and the NMR signal intensities scale with the layer thickness, as shown in Figure 5. At this point we want to emphasize that the applied procedure leads to the "final" NMR spectra $I_{0}(f)$, having comparable intensities in all studied samples, despite the fact that the experimentally recorded intensities and the used $h_{1}$ amplitudes differ by more than one order of magnitude (Figure 4).

Two clearly resolved lines with a Gaussian-like shape are present in each spectrum in Figure 5. Interestingly their respective intensity ratio depends on the value of tetragonal lattice distortion. To simplify further discussion we label the line at a central frequency of $376 \mathrm{MHz}$ as $\mathbf{f}_{0}$, while the other line, around $330 \mathrm{MHz}$, as $\mathbf{f}_{\mathbf{1}}$.

It is well established that the $\mathbf{f}_{\mathbf{0}}$ line in ferromagnetic, metallic and virtually cubic LSMO, is due to $\mathrm{Mn}$ ions in a mixed valence state $\mathrm{Mn}^{3+/ 4+}$ created by very fast $\left(\tau<10^{-9} \mathrm{~s}\right)$ electron interchange between $\mathrm{Mn}^{3+}$ and $\mathrm{Mn}^{4+}$ ions participating in the three dimensional DE coupling (3D DE). Therefore $\mathrm{Mn}^{3+/ 4+}$ ions giving rise to this line are contributing to the magnetization and to electric conduction $[16,17,18,19,46,47,48,49,50,51]$. More precisely, the observed frequency $f_{0} \approx 376 \mathrm{MHz}$ is in good agreement with the expected frequency value for a half-doped manganite [51]. Now, we focus on the origin of the line $\mathbf{f}_{1}$. We first consider the differences in the intensities of the $f_{0}$ and $\mathbf{f}_{1}$ NMR lines in the three panels of Figure 5. Comparing the spectra in the middle (non-strained film on LSAT) and the top (tensile strained film on STO) panels one can readily notice that the intensity of $f_{0}$ line has dramatically decreased whereas that of $\mathbf{f}_{\mathbf{1}}$ has increased in the strained film. In fact the intensity ratio between $\mathbf{f}_{1}$ and $\mathbf{f}_{0}$ has been practically interchanged when going from LSMO//LSAT to LSMO//STO. Reduction of the intensity of the $\mathbf{f}_{0}$ line is consistent with the fact that this line is a fingerprint of the ferromagnetic-metallic fraction in the sample, and the observation (Figure 3(c)) that the magnetization of the strained film (LSMO//STO) is much reduced [10]. Figure 6 illustrates the relationship between the intensities of the $f_{0}$ an $f_{1}$ lines and the sample's magnetization as derived from magnetic (SQUID) measurements [10]. By the same token, the $f_{1}$ line cannot be attributed to a ferromagnetic fraction of the sample characterized by a different ${ }^{55} \mathrm{Mn}$ NMR frequency. Indeed, using the $f(x)$ expression derived in [51] to describe the hole concentration dependence of the ${ }^{55} \mathrm{Mn}$ 
NMR frequency in DE governed ferromagnetic manganites, the observed $\mathbf{f}_{\mathbf{1}} \approx 330-336 \mathrm{MHz}$, would correspond to an unrealistic hole concentration $(x \approx 1)$. Therefore, neither the intensity evolution of the $\mathbf{f}_{1}$ line nor its frequency, allow to assign $\mathbf{f}_{1}$ line to a ferromagnetic contribution.

Focusing now on the comparison of the middle and bottom panels in Fig. 5, it is clear that the total intensity under the $\mathbf{f}_{0}$ line is also largely reduced in compressively strained films (LSMO//YAO). This is also consistent with observed reduction of the magnetization (Figures $3(c)$ and 6). One also notices that the relative fraction of $\mathbf{f}_{1}$ and $\mathbf{f}_{0}$ is almost preserved.

Understanding these variations demands a critical revision of applicability of the conventional wisdom used to interpret NMR data of manganites to the present case of the orbitally ordered $\mathrm{La}_{1 / 2} \mathrm{Sr}_{1 / 2} \mathrm{MnO}_{3}$. It is commonly reported that in the optimally doped manganites $\left(\operatorname{La}_{2 / 3} \mathrm{~A}_{1 / 3} \mathrm{MnO}_{3}\right.$, $\mathrm{A}=\mathrm{Ca}, \mathrm{Sr}$ ), the NMR spectra display a similar shape than those of Fig. 5 , with two resonance lines at similar frequencies. The most intense resonance line $\left(\mathbf{f}_{0}\right)$ located at high frequency is related to the 3D DE $\mathrm{Mn}^{3 / 4+}$ ions. The resonance observed at lower frequency is attributed to localized $\mathrm{Mn}^{4+}$ ferromagnetic ions, formed due to the charge localization $[16,19,47,48,49,50]$ as sketched in Figure $7(a)$. Consistently, in that scenario the sample magnetization scales well with the total intensity under the NMR spectra, including both lines assigned to the itinerant $\mathrm{Mn}^{3 / 4+}$ and to the localized $\mathrm{Mn}^{4+}$ ions $[16,48,49,50]$. As clearly illustrated by data in Figure 6 , the situation is different in the present $\mathrm{La}_{1 / 2} \mathrm{Sr}_{1 / 2} \mathrm{MnO}_{3}$ films and a radically different scenario should be envisaged to account for the origin of the $\mathbf{f}_{\mathbf{1}}$.

The starting point for the discussion is the data in Figure 5. It indicates that the electronic configuration of $3 \mathrm{~d}\left(\mathrm{e}_{\mathrm{g}}\right)$ state of a fraction of $\mathrm{Mn}$ ions contributing to the isotropic double exchange interaction and represented in NMR spectrum by the $f_{0}$ line, has been changed due to presence of an epitaxial strain.

In order to trace this change let us consider how strain can influence the hyperfine field experienced by ${ }^{55} \mathrm{Mn}$ nuclei in manganites. It is well known that for a octahedraly coordinated $\mathrm{Mn}^{3+}\left(3 \mathrm{~d}^{4}\right)$ ions with an orbital $3 \mathrm{~d}\left(\mathrm{e}_{\mathrm{g}}\right)$ doublet as a ground state, the hyperfine field can be strongly anisotropic $[52,53]$ in contrast to the case of $\mathrm{Mn}^{4+}\left(3 \mathrm{~d}^{3}\right)$ ions where an orbital singlet state $3 d\left(t_{2 g}\right)$ gives rise to isotropic hyperfine field. Indeed, in case of $\mathrm{Mn}^{3+}$ ions, an electron located in $3 d\left(e_{g}\right)$ orbitals $\left(x^{2}-y^{2}\right.$, $\left.3 z^{2}-r^{2}\right)$ will create a dipolar hyperfine field $\left(\mathbf{H}_{\text {dip }}\right)$ due to its own spin dipole moment, that will add to an isotropic hyperfine field component due to the Fermi contact interaction $\left(\mathbf{H}_{\text {con }}\right)$. The dipolar hyperfine field is strongly anisotropic and its value depends on the symmetry of the $3 \mathrm{~d}\left(\mathrm{e}_{\mathrm{g}}\right)$ orbital occupied by an electron and the orientation of the spin moment. It turns out that for each orientation of the spin moment, the relation $\mathrm{H}_{\text {dip }}\left(x^{2}-y^{2}\right)=-H_{\text {dip }}\left(3 z^{2}-r^{2}\right)$ holds valid and $H_{\text {dip }}\left(x^{2}-y^{2}\right)$ $(\mathbf{S} / / \mathrm{xy})=(1 / 2) * \mathrm{H}_{\text {dip }}\left(3 z^{2}-r^{2}\right)(\mathbf{S} / / z)$ (where $\mathbf{S}$ indicates either in-plane $(x y)$ or out-of-plane $(z)$ electron spins) are always parallel $[52,54]$.

In case of a cubic symmetry, the $3 \mathrm{~d}\left(\mathrm{e}_{\mathrm{g}}\right)$ state of $\mathrm{Mn}^{3+}$ ions is degenerated and thus the probability of an electron to have the symmetry of one of the $e_{g}$ orbitals is identical. Consequently, the dipolar hyperfine field due to $3 \mathrm{~d}\left(\mathrm{e}_{\mathrm{g}}\right)$ electrons vanishes for any orientation of the electron spin and only the isotropic Fermi contact hyperfine field remains. In contrast, the situation changes when the local symmetry is lowered from cubic to tetragonal, resulting in the elongation or compression of $\mathrm{MnO}_{6}$ octahedra, favoring $\left(3 z^{2}-r^{2}\right)$ orbital occupation for compressive strain or favoring the $\left(x^{2}-y^{2}\right)$ occupation for tensile strain, as confirmed by the XLD data shown in a previous section. In any event, one of the $3 \mathrm{~d}\left(\mathrm{e}_{\mathrm{g}}\right)$ orbitals lowers its energy and becomes more populated and the condition for a mutual cancelation of the dipolar hyperfine fields due to spin of $3 \mathrm{~d}\left(\mathrm{e}_{\mathrm{g}}\right)$ electrons is no longer fulfilled. The hyperfine field for $\mathrm{Mn}^{3+}$ ions becomes an average between fields of $\mathrm{Mn}^{3+}$ with $3 \mathrm{~d}\left(\mathrm{e}_{\mathrm{g}}\right)$ electron on $\left(\mathrm{x}^{2}-\mathrm{y}^{2}\right)$ orbital and $\mathrm{Mn}^{3+}$ with $3 \mathrm{~d}\left(\mathrm{e}_{\mathrm{g}}\right)$ electron on $\left(3 \mathrm{z}^{2}-\mathrm{r}^{2}\right)$ orbital weighted by their relative population, itself modulated by the strain. Such a scenario, in case of LSMO tensely strained thin film favoring in-plane $3 d\left(e_{g}\right)$ orbitals $\left(x^{2}-y^{2}\right)$ and for the in-plane orientation of magnetic moments, leads to a reduction of the hyperfine field $[52,54]$ experienced by $\mathrm{Mn}^{3 / 4+}$ ions due to the opposite 
orientation of the Fermi contact $\left(\mathbf{H}_{\text {con }}\right)$ and the dipolar $\left(\mathbf{H}_{\text {dip }}\right)$ hyperfine fields, as sketched in Figure 7(b).

Based on the above relation between strain and hyperfine field experienced by ${ }^{55} \mathrm{Mn}$ nuclei, the presence of the $\mathbf{f}_{1}$ line in all spectra recorded from LSMO films is interpreted as a signature of the electron orbital preferential occupancy and an associated anisotropic dipolar contribution. Observation of $\mathbf{f}_{\mathbf{1}}<\mathbf{f}_{\mathbf{0}}$, indicating a preferential occupancy of $x^{2}-y^{2}$ orbitals (Figure $\mathbf{7}(\mathbf{b})$ ) and in-plane spins, is consistent with the presence of an A-type antiferromagnetic phase (A-AF) displaying ferromagnetic order in planes but with a prevailing antiferromagnetic coupling between planes. Therefore, in the experiments reported here the observation of $f_{1}$ line in ${ }^{55} \mathrm{Mn} N M R$ spectra of LSMO//STO is regarded as a signature of A-AF type local ordering in the studied samples. We note in passing, that the presence of the $f_{1}$ line in the NMR spectra for nominally non strained film (LSMO//LSAT), as well as of LSMO bulk sample, can be taken as a microscopic evidence of the lowtemperature spontaneous transformation of LSMO from cubic to orthorhombic (A-type) phase as derived from neutron diffraction experiments on bulk samples [55].

In view of the proposed modification of $\mathrm{Mn}^{3+}$ hyperfine field triggered by strain, it could be expected that the spectrum recorded for compressively strained LSMO//YAO sample would show a different resonance line at $\mathbf{f}_{1}{ }_{1}<f_{0}$, and $f^{\prime}{ }_{1}$ would fulfill a relation $\left|f_{0}-f_{1}\right|=(1 / 2)\left|f_{0}-f^{\prime}{ }_{1}\right|$, because compressive strain favors a different imbalance of the dipolar hyperfine field component due to a preference for $\left(3 z^{2}-r^{2}\right)$ orbital occupation (Figure 7(c)), as indicated above, [52,54]. However, this would correspond to a C-type orbital ordering and an associated C-AF ordering, with spins pointing perpendicular to the film plane (as confirmed by the XLD data reported above). This 3D antiferromagneticaly coupled structure cannot be easily excited by the very small in-plane $h_{1}$ field used and thus it cannot be observed in the corresponding NMR spectrum. On the other hand, the observation in LSMO//YAO film, of a resonance line at $\mathbf{f}_{\mathbf{1}}<\mathbf{f}_{\mathbf{0}}$ may seem at first sight surprising. However, it has to be recalled that the analysis of a lattice mismatch in case of YAO substrate [10] indicates a partial reduction of strain and a relaxed structure. Therefore, this observation can be regarded as the evidence of a nonhomogeneously relaxed state of the LSMO//YAO films, with the strain-free or almost strain-free regions of the LSMO//YAO film, giving rise to the NMR spectrum characteristic for the unstrained LSMO//LSAT film.

These observations also imply that each of the two forms of antiferromagnetic order (A-AF and C-AF) influence the restoring field $\left(\mathrm{H}_{\text {res }}\right)$ of a phase separated material in a different way. This aspect of the effect of strain on the restoring field can be traced by inspecting the changes of the restoring field for the LSMO films on LSAT, STO and YAO substrates. Indeed, systematic measurements of the restoring field, show that in the frequency region of interest, from $280 \mathrm{MHz}$ to $440 \mathrm{MHz}$, the corresponding values are $\approx 2$ Oe (LSAT), $\approx 10$ Oe (STO) and $\approx 30$ Oe (YAO) (see Figure 4 ), which confirm the magnetic hardening associated with the presence of A-type and C-type spin orderings. On the other hand, microstructural effects such as dislocations formed when strain relaxation occurs (case of YAO) may also contribute to increasing magnetic stiffness of the film, by reinforcing magnetic domain wall pinning. Since our NMR experiment has been carried out without external field i.e. in a demagnetized state, some kind of a domain structure must be present in all samples. Consequently, the experimentally determined restoring field should be analyzed in the context of all possible domain wall excitations leading to the NMR enhancement $\eta$ which can be active in these phase separated materials.

Finally, we recall that NMR signal intensity is proportional to the number of nuclei resonating at a given frequency, and the integrated spectrum intensity is proportional to their total number. Figure 6 presents the NMR spectrum intensities: total $\left(f_{0}+f_{1}\right)$ and decomposition split into $f_{0}$ and $f_{1}$ contributions as a function of magnetization, measured by SQUID. This comparison shows that not only both techniques reveal a reduction of the ferromagnetic component by strain, but a proportionality between magnetization detected by SQUID and the intensity of the ferromagnetic 
fraction represented by $f_{0}$ line in NMR spectra is observed (circles in Figure 6), as a response to the increasing tetragonal lattice distortion due to the strain. The fraction of $\mathrm{Mn}$ ions giving rise to the $\mathbf{f}_{1}$ line (thus corresponding to the antiferromagnetic A-type phase) is not contributing to magnetization, whereas it is detected in the NMR experiment. We note that NMR signal from Mn ions can be excited under the small driving $h_{1}$ field as far as their magnetic moments can be brought to oscillation generating in turn an oscillating transverse component of the hyperfine field and an effective field $(1+\eta) h_{1}$ acting on Mn nuclear spins. In a single-phase antiferromagnet, this is not possible and thus AF materials do no respond to $h_{1}$. However, A-type antiferromagnets have in-plane ferromagnetically aligned spins, which coexist with ferromagnetic phases. Therefore, exchange coupling and thus coupled oscillating responses to $\boldsymbol{h}_{1}$, giving rise to an enhanced spin-echo NMR signal, can be expected in such case [53]. Consistently, the smaller value of the total NMR intensity for YAO is due to a missing intensity related to $\mathrm{Mn}$ atoms involved in C-type antiferromagnetic phase.

In summary, the present ${ }^{55} \mathrm{Mn}$ NMR study provides clear evidence of strain-induced orbital ordering and concomitant phase separation into A-type and C-type antiferromagnetic orders that coexist with residual ferromagnetic phases in LSMO.

\section{SUMMARY AND CONCLUSIONS}

We have reported an extensive study of the magnetic and orbital order of strained epitaxial halfdoped manganite films by studying the dependence of X-ray absorption with linear and circular light polarization on the tetragonal distortion of the films. LSMO films show an almost linear dependence of the orbital occupancy with the tetragonal distortion, indicating a Jahn-Teller-like mechanism for the stabilization of $x^{2}-y^{2}\left(3 z^{2}-r^{2}\right)$ states for tensile (compressive) strain. Surface symmetry breaking effects are also shown to be relevant by contributing to a larger occupancy of $3 z^{2}-r^{2}$ states, added to the strain induced occupancy.

The coupling between orbital and spin degrees of freedom is manifested in LSMO samples by the stabilization of an AF phase with in-plane spin ordering for tensile strain, which is in accordance with the predicted A-AF phase promoted by the $x^{2}-y^{2}$ orbital order, which favours the delocalization of electrons in the xy plane and reduces the hopping along the c-axis, thus resulting in a (in-plane) metallic character. Both the AF and metallic character of these films are corroborated by transport and magnetic measurements [10]. On the other hand, compressive strain is shown to favour an AF phase with spin axis pointing in the c-axis direction, as expected from the predicted C-AF phase, coupled to the $3 z^{2}-r^{2}$ orbital order. This phase presents a strongly insulating character, also observed in macroscopic measurements [10]. Finally, on well matched substrates, where strain does not largely break the $\mathrm{e}_{\mathrm{g}}$ states degeneracy, a ferromagnetic and metallic phase is observed, both by $\mathrm{x}$-ray absorption and macroscopic measurements. The phase diagram shown in Figure 8 summarizes these findings.

It is found that the orbital occupancy in the narrower band LCMO HD manganite is less affected by Jahn-Teller-like unit cell distortions, as XLD shows that the induced modification in the XLD signal is less sensitive to the tetragonality ratio (c/a) in LCMO for films under compressive or moderate tensile strain. It has been argued that rigid rotation of the $\mathrm{Mn}$ coordination polyhedra, rather than Jahn-Teller like distortions, combined with the genuine $3 z^{2}-r^{2}$ surface contribution may account for these observations. In contrast, tensile strained samples, show a strong dichroism indicative of a strongly modified orbital order, probably connected to the predicted CE-phase. Indeed low temperature dichroism measurements point to a AF order with in-plane spin axis for these samples. On the other hand, measurements on compressive strained samples suggest a phase coexistence of $\mathrm{F}$ and C-AF phases. The strong predominance of AF phases in LCMO is also consistent with 
macroscopic magnetic measurements [10], which also confirm the insulating character of these samples (Figure 8).

${ }^{55} \mathrm{Mn}$ NMR data has brought consistent evidence of a suppression of the ferromagnetic phase under strain. Moreover NMR has provided a new insight into orbital ordering by exploiting the sensitivity of the electron spin dipolar hyperfine field on $3 \mathrm{~d}\left(\mathrm{e}_{\mathrm{g}}\right)$ orbital occupancy which give rise to distinguishable resonance lines in the NMR spectrum for electrons at $x^{2}-y^{2}$ and $3 z^{2}-r^{2}$ states. By itself, this finding may open new perspective on using NMR techniques to explore orbital occupancy in solids.

In conclusion, we have established a clear connection between the magnetic and transport properties of half-doped manganite films and the electronic and magnetic structure characteristics giving rise to the observed macroscopic properties. From the perspective of implementing HD manganite layers in ferroelectric tunnel junctions, aiming at inducing controlled metal-insulator transitions, our results indicate that even in the cases of rather thick films as those reported here (20 $\mathrm{nm}$ ) strain and interface effects have dramatic consequences on charge localization, orbital and spin ordering, accompanied by indications of phase separation. This complex interplay, which probably is reinforced in ultrathin films as required in tunnel devices, suggests that it may be difficult to obtain high-yield successful processing of electroresistive junctions. Alternative approaches may be envisioned by selecting materials or compositions less prone to phase separation. 


\section{Figure captions:}

Table 1. Calculated lattice mismatch between bulk pseudo-cubic lattice parameters of LSMO, LCMO and substrates, and c/a ratio measured in the films.

FIG. 1. Normalized X-ray absorption spectra obtained with in-plane ( $\mathrm{l}_{a b}$ - solid line) and out-of-plane $\left(\mathrm{I}_{\mathrm{C}}\right.$ - dashed line) $\mathrm{x}$-ray polarization of LSMO (a) and LCMO (b) samples, measured at $300 \mathrm{~K}$; bottom of panel (a): difference XAS spectra between LSAT and DSO samples and simulated $\mathrm{Mn}^{2+}$ spectrum; resultant XLD spectra $\left(I_{a b}-I_{C}\right)$ of LSMO (c) and LCMO (b) samples (shaded areas indicate region of integration as indicated in the text).

FIG.2. Integrated area under XLD as a function of c/a ratio for LSMO (solid squares) and LCMO (empty circles) films, grown on DSO (black symbols), STO (blue), LSAT (green), YAO (red) and LAO (orange) substrates. Lines correspond to linear fittings of $I_{X L D}$ (c/a) for all LSMO samples (solid line) and for all LCMO samples except film on STO (dashed line).

FIG.3. XMCD spectra of LSMO (a) and LCMO (d) samples measured at 5 and $50 \mathrm{~K}$ respectively (shaded areas indicate region of integration after subtraction of $\mathrm{Mn}^{2+}$ contribution as indicated in the text); XMLD (XLD(5 K, $50 \mathrm{~K})$ - XLD (300 K)) spectra of LSMO (b) and LCMO (e) samples; normalized integrated area under XMCD at $L_{2}$ edge (left axis, solid squares, solid line) and intensity difference between first and second peak of XMLD at $L_{2}$ edge (right axis, empty circles, dashed line) for LSMO (c) and LCMO (f) samples as a function of c/a ratio. All measurements taken with applied field $B=2 T$.

FIG.4. Spin echo intensity in LSMO (36nm) thin films at $376 \mathrm{MHz}$ (films on LSAT, YAO) and $330 \mathrm{MHz}$ (film on STO) as a function of r.f. field strength expressed in the units of restoring field $\left(\beta \mathrm{h}_{1}\right)$. Dashed curves are fittings to log-normal function. Vertical arrows indicate $h_{1 o p t}$ corresponding to $H_{\text {rest }}$, horizontal arrows indicate maximum signal intensity $\mathrm{I}_{\mathrm{opt}}$ corresponding to $\mathrm{I}_{0}$.

FIG.5. ${ }^{55} \mathrm{Mn}$ NMR spectra recorded at $4.2 \mathrm{~K}$ from $20 \mathrm{~nm}$ and $36 \mathrm{~nm}$ LSMO films grown on STO (a) LSAT (b) and YAO (c). ${ }^{55} \mathrm{Mn}$ NMR spectrum recorded from bulk LSMO is also shown in the middle panel (b). Arrow lines indicate the frequencies $\mathbf{f}_{0}$ and $\mathbf{f}_{1}$. All ${ }^{55} \mathrm{NMR}$ spectra have been corrected for enhancement factor following the Panissod procedure [23] and the spectrum intensity at each frequency point is proportional - with a common proportionality factor for all spectra taking into account samples mass - to number of nuclei ( $\mathrm{Mn}$ atoms) characterized by this resonance frequency.

FIG.6. ${ }^{55} \mathrm{Mn}$ NMR spectrum intensity registered at $4.2 \mathrm{~K}$ as a function of magnetization in LSMO $20 \mathrm{~nm}$ (solid symbols) and $36 \mathrm{~nm}$ (empty symbols) thin films for LSAT, YAO and STO substrates. NMR intensity has been obtained by integrating spectra in $300-420 \mathrm{MHz}$ range $\left(f_{0}+f_{1}\right.$ lines) (black symbols), 300-350 MHz range ( $f_{1}$ line, yellow symbols), 350-420 MHz range ( $f_{0}$ line, purple symbols) and normalized to layer thickness for comparison of 20 and $36 \mathrm{~nm}$ thick layers.

FIG.7. (a) Sketch of electronic phase separated $\mathrm{Mn}^{3+/ 4+}$ and $\mathrm{Mn}^{4+}$ ferromagnetic regions in optimally doped $\mathrm{La}_{2 / 3} \mathrm{Sr}_{1 / 3} \mathrm{MnO}_{3}$ as inferred from ${ }^{55} \mathrm{Mn}$ NMR spectroscopy $[16,18,19,48,49,50]$. (b) and (c) sketches of the $\left(x^{2}-y^{2}\right)$ and $\left(3 z^{2}-r^{2}\right)$ orbitals and associated A-type and C-type AF spin ordering and the corresponding dipolar hyperfine field $\left(\mathrm{H}_{\text {dip }}\right)$ (blue/red arrows) and hyperfine Fermi contact field $\left(\mathrm{H}_{\text {con }}\right)$ (black arrows) acting on the ${ }^{55} \mathrm{Mn}$ nuclei [54].

FIG.8. Strain phase diagram of half-doped manganite films, indicating the orbital and magnetic phase derived from experiments and theoretical calculations. Shaded areas indicate regions where samples show metallic character.

\section{Acknowledgments:}

This work has been partially supported by grants from the Spanish Government (MAT2014-56063C2-1R and SEV-2015-0496), the Catalan Government (2014 SGR 734), the Italian Government (MIURProgetto Premiale "Materiali e disposivi magnetici e superconduttivi per sensoristica e ICT"), the 
National Sciences Centre of Poland (DEC 2011/03/B/ST3/02368) and the EU FP7 Program (REGPOTCT-2013-316014 (EAgLE)). 
LSMO

\begin{tabular}{lccccc}
\cline { 2 - 3 } \cline { 5 - 6 } Substrate & $\delta(\%)$ & $\mathrm{c} / \mathrm{a}$ & & $\delta(\%)$ & $\mathrm{c} / \mathrm{a}$ \\
\hline DSO & +2.13 & 0.958 & & +2.87 & 0.955 \\
\hline STO & +1.22 & 0.973 & & +1.96 & 0.957 \\
\hline LSAT & +0.31 & 0.990 & & +1.04 & 0.975 \\
\hline YAO & -3.58 & 1.007 & & -2.87 & 1.017 \\
\hline LAO & -1.71 & 1.043 & & -0.99 & 1.023
\end{tabular}

Table 1. 


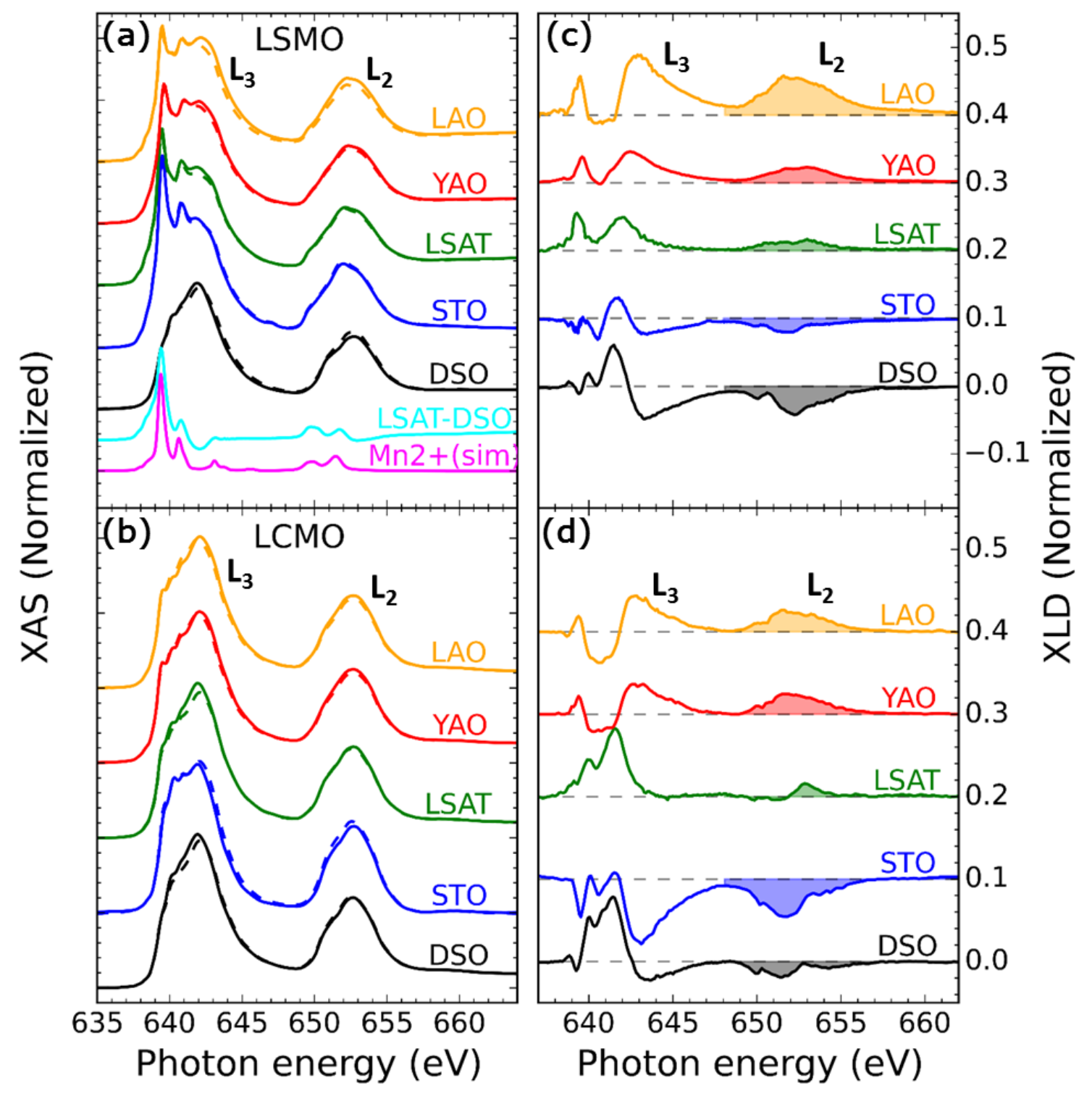

Figure 1. 


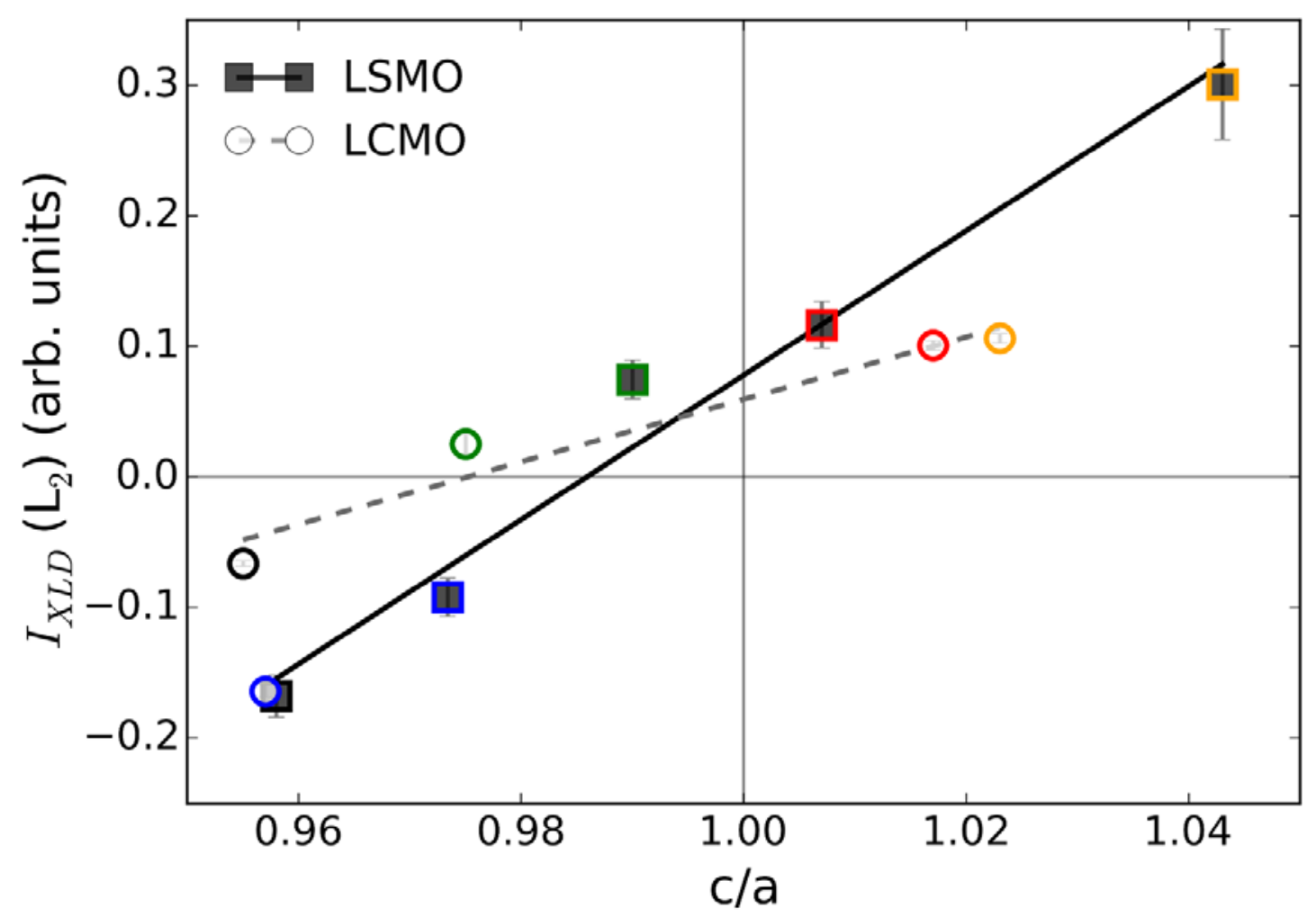

Figure 2. 

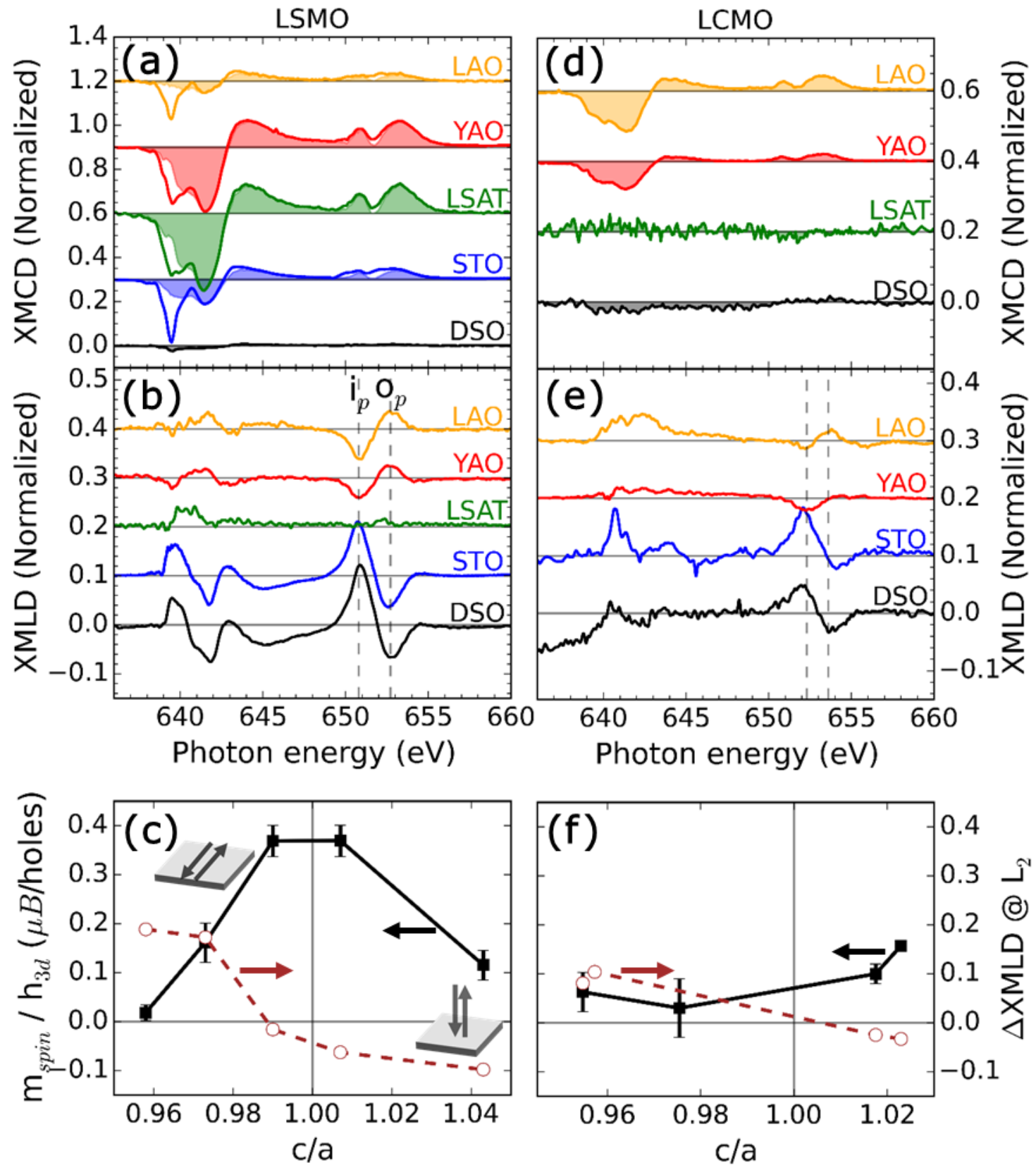

Figure 3. 


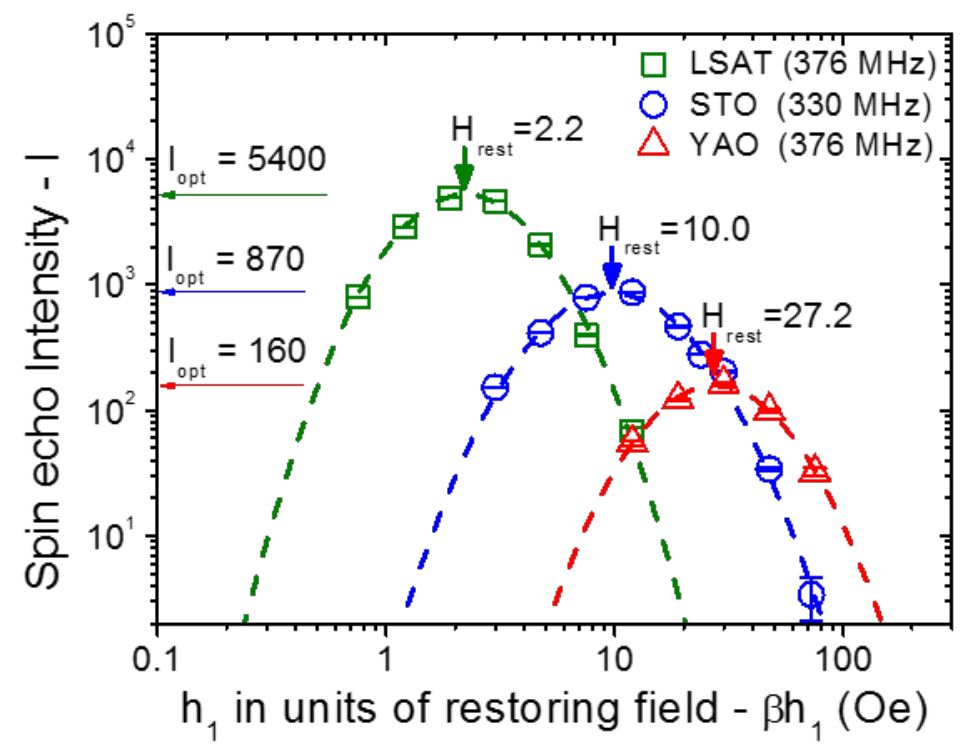

Figure 4. 


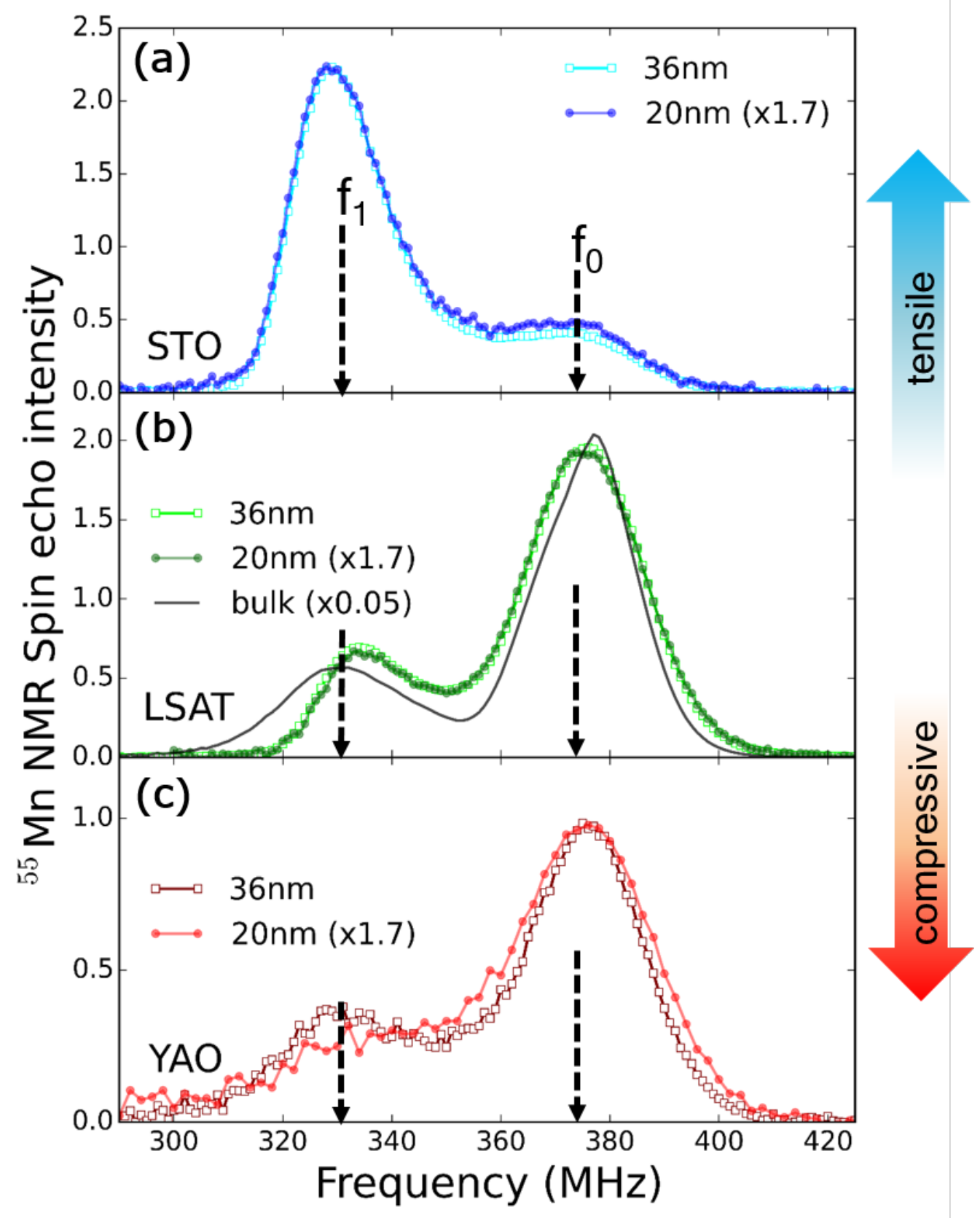

Figure 5. 


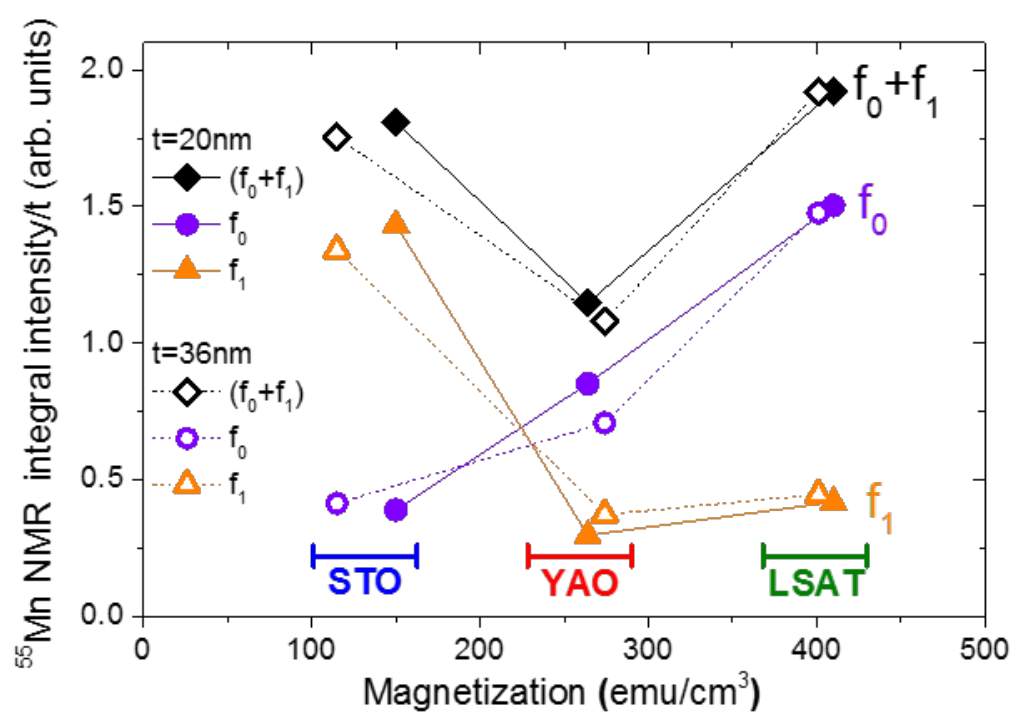

Figure 6. 
a)

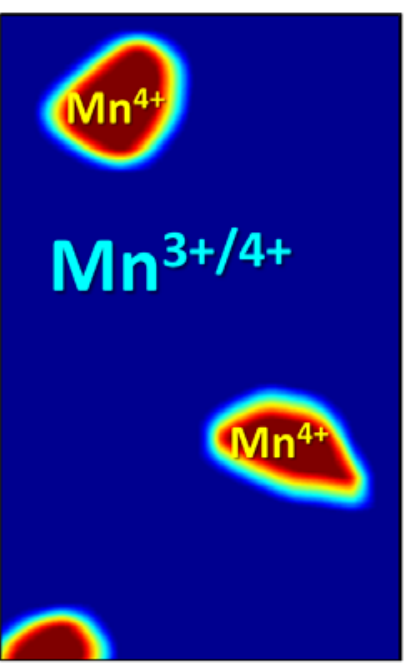

b)

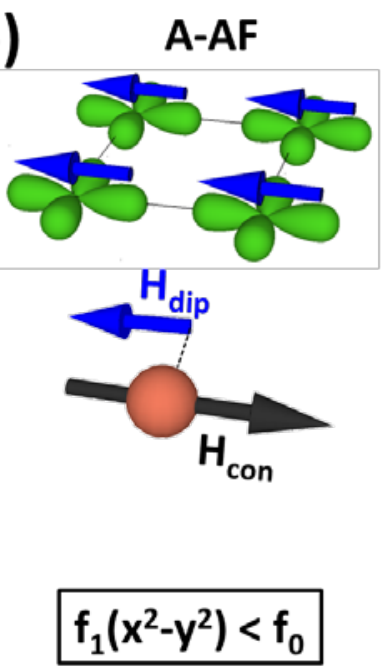

c) C-AF

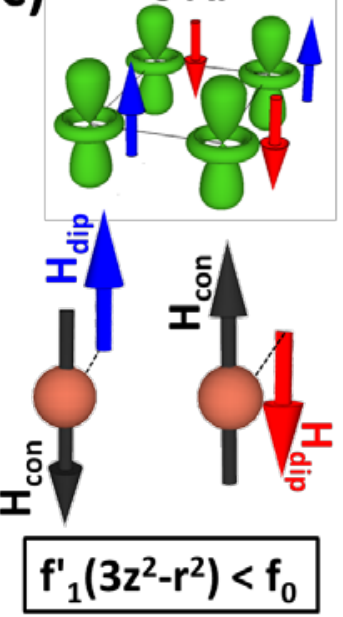

Figure 7. 


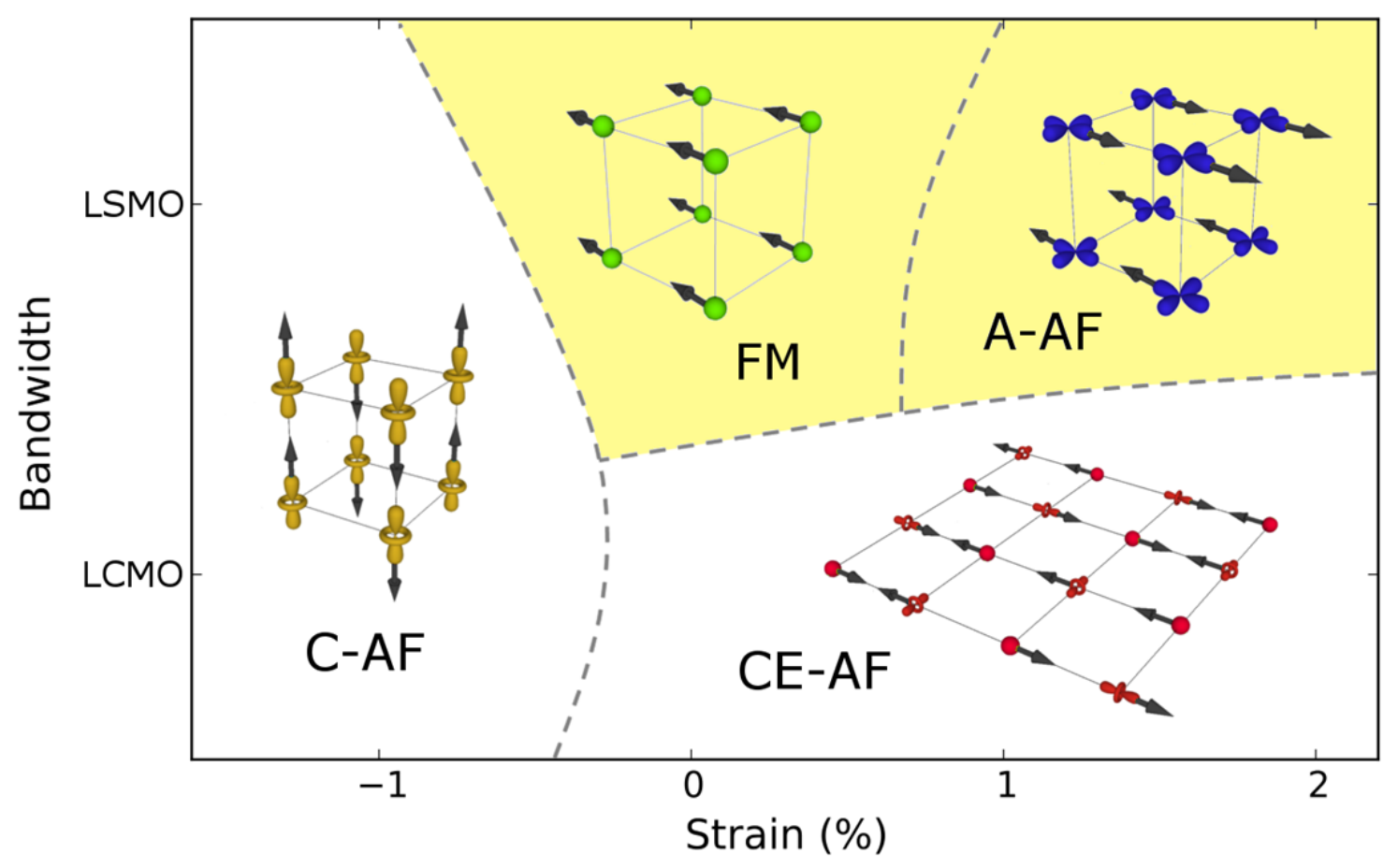

Figure 8. 
${ }^{1}$ F. Matsukura, Y. Tokura, and H. Ohno, Control of magnetism by electric fields, Nat. Nanotechnol. 10, 209 (2015).

2 H.J. A. Molegraaf, J. Hoffman, C. A. F. Vaz, S. Gariglio, D. van der Marel, C.H. Ahn, and J.-M. Triscone, Magnetoelectric effects in complex oxides with competing ground states, Adv. Mater. 21, 3470 (2009).

${ }^{3}$ D. Preziosi, M. Alexe, D. Hesse, and M. Salluzzo, Electric-field control of the orbital occupancy and magnetic moment of a transition-metal oxide, Phys. Rev. Lett. 115, 157401 (2015).

${ }^{4}$ P. Schiffer, A. P. Ramirez, W. Bao, and S.-W. Cheong, Low temperature magnetoresistance and the magnetic phase diagram of $\mathrm{La}_{1-x} \mathrm{Ca}_{x} \mathrm{MnO}_{3}$, Phys. Rev. Lett. 75, 3336 (1995).

${ }^{5}$ V. Garcia, M. Bibes, L. Bocher, S. Valencia, F. Kronast, A. Crassous, X. Moya, S. Enouz-Vedrenne, A. Gloter, D. Imhoff, C. Deranlot, N.D. Mathur, S. Fusil, K. Bouzehouane, and A. Barthélémy, Ferroelectric control of spin polarization, Science 327, 1106 (2010).

${ }^{6}$ A. Gruverman, D. Wu, H. Lu, Y. Wang, H.W. Jang, C.M. Folkman, M.Y. Zhuravlev, D. Felker, M. Rzchowski, C.B. Eom, and E.Y. Tsymbal, Tunneling electroresistance effect in ferroelectric tunnel junctions at the nanoscale, Nano Lett. 9, 3539 (2009).

${ }^{7}$ L. Jiang, W.S. Choi, H. Jeen, S. Dong, Y. Kim, M.-G. Han, Y. Zhu, S. V Kalinin, E. Dagotto, T. Egami, and H.N. Lee, Tunneling electroresistance induced by interfacial phase transitions in ultrathin oxide heterostructures, Nano Lett. 13, 5837 (2013).

${ }^{8}$ G. Radaelli, D. Gutiérrez, F. Sánchez, R. Bertacco, M. Stengel, and J. Fontcuberta, Large room-temperature electroresistance in dual-modulated ferroelectric tunnel barriers, Adv. Mater. 27, 2602 (2015).

${ }^{9}$ Y.W. Yin, J.D. Burton, Y.-M. Kim, A Y. Borisevich, S.J. Pennycook, S.M. Yang, T.W. Noh, A. Gruverman, X.G. Li, E.Y. Tsymbal, and Q. Li, Enhanced tunnelling electroresistance effect due to a ferroelectrically induced phase transition at a magnetic complex oxide interface, Nat. Mater. 12, 397 (2013).

${ }^{10}$ D. Gutiérrez, G. Radaelli, F. Sánchez, R. Bertacco, and J. Fontcuberta, Bandwidth-limited control of orbital and magnetic orders in half-doped manganites by epitaxial strain, Phys. Rev. B, 89, 075107 (2014).

${ }^{11}$ Y. Tokura, Orbital physics in Transition-metal oxides, Science 288, 462 (2000)

${ }^{12}$ A. Baena, L. Brey, and M. J. Calderón, Effect of strain on the orbital and magnetic ordering of manganite thin films and their interface with an insulator, Phys. Rev. B 83, 064424 (2011).

${ }^{13}$ Z. Fang, I. V. Solovyev, and K. Terakura, Phase diagram of tetragonal manganites, Phys. Rev. Lett. 84, 3169 (2000).

${ }^{14}$ Y. Konishi, Z. Fang, M. Izumi, T. Manako, M. Kasai, H. Kuwahara, M. Kawasaki, K. Terakura, and Y. Tokura, Orbital-state-mediated phase-control of manganites, J. Phys. Soc. Jpn. 68, 3790 (1999).

${ }^{15}$ J. C. Loudon, N. D. Mathur, and P. A. Midgley, Charge ordered ferromagnetic phase in $\mathrm{La}_{0.5} \mathrm{Ca}_{0.5} \mathrm{MnO}_{3}, \mathrm{Nature}$ 420, 797 (2002).

${ }^{16}$ a) M. Bibes, L. Balcells, S. Valencia, J. Fontcuberta, M. Wojcik, E. Jedryka, S. Nadolski, Nanoscale multiphase separation at $\mathrm{La}_{2 / 3} \mathrm{Ca}_{1 / 3} \mathrm{MnO}_{3} / \mathrm{SrTiO}_{3}$ interfaces, Phys. Rev. Lett., 87, 067210 (2001); b) M. Bibes, S. Valencia, B. Martinez, J. Fontcuberta, M. Wojcik, S. Nadolski, and E. Jedryka, Charge trapping in optimally doped epitaxial manganite thin films, Phys. Rev. B, 66, 134416 (2002).

${ }^{17}$ G. Matsutomo, Study of $\left(\mathrm{La}_{1-\mathrm{x}} \mathrm{Ca}_{\mathrm{x}}\right) \mathrm{MnO}_{3}$. I. Magnetic structure of $\mathrm{LaMnO}_{3}$ J. Phys. Soc. Jpn., 29, 606 (1970).

${ }^{18}$ M. M. Savosta, P. P. Novák, Two-phase character of metallic ferromagnetism in manganites, Phys. Rev. Lett 87, 137204 (2001).

${ }^{19}$ G. Allodi, R. De Renzi, G. Guidi, F. Licci, and M. W. Pieper, Electronic phase separation in lanthanum manganites: Evidence from ${ }^{55} \mathrm{Mn}$ NMR, Phys. Rev. B 56, 6036 (1997). 
${ }^{20}$ C.A.F. Vaz, J.A. Moyer, D.A. Arena, C.H. Ahn, and V.E. Henrich, Reversible modulation of orbital occupations via an interface- induced polar state in metallic manganites Phys. Rev. B 90, 024414 (2014).

${ }^{21}$ The strongly insulating character of LCMO films at low temperatures impeded the TEY measurements at $\mathrm{T}<50 \mathrm{~K}$

${ }^{22}$ W. B. Wu, D. J. Huang, G. Y. Guo, H.-J. Lin, T. Y. Hou, C. F. Chang, C. T. Chen, A. Fujimori, T. Kimura, H. B. Huang, A. Tanaka, and T. Jo, Orbital polarization of $\mathrm{LaSrMnO}_{4}$ studied by soft X-ray linear dichroism, J. Electron Spectros. Relat. Phenomena 641, 137, (2004).

${ }^{23}$ P. Panissod, M. Malinowska, E. Jedryka, M. Wojcik, S. Nadolski, M. Knobel, and J.E. Schmidt, Inhomogeneous structure and magnetic properties of granular $\mathrm{Co}_{10} \mathrm{Cu}_{90}$ alloys, Phys. Rev. B 63, 014408 (2000).

${ }^{24} \mathrm{Mn}^{2+}$ XAS spectrum simulated with CTM4XAS software for a cubic crystal field with 10Dq=0.5 eV

${ }^{25}$ E. Stavitski and F. De Groot, The CTM4XAS program for EELS and XAS spectral shape analysis of transition metal L edges, Micron Oxford England 41, 687, (2010)

${ }^{26}$ M. de Jong, I. Bergenti, V. Dediu, M. Fahlman, M. Marsi, and C. Taliani, Evidence for $\mathrm{Mn}^{2+}$ ions at surfaces of $\mathrm{La}_{0.7} \mathrm{Sr}_{0.3} \mathrm{MnO}_{3}$ thin films, Phys. Rev. B 71, 014434 (2005).

${ }^{27}$ S. Valencia, A. Gaupp, W. Gudat, L. Abad, L. Balcells, A. Cavallaro, B. Martínez, and F. Palomares, Mn valence instability in $\mathrm{La}_{2 / 3} \mathrm{Ca}_{1 / 3} \mathrm{MnO}_{3}$ thin films, Phys. Rev. B 73, 104402 (2006).

${ }^{28}$ D. Pesquera, Strain and interface induced electronic states in transition metal oxides, PhD Thesis, Universitat Autònoma de Barcelona (Spain) (2014) (https://www.educacion.gob.es/teseo/mostrarRef.do?ref = 1110837\#)

29 A. V. Boris, Y. Matiks, E. Benckiser, A. Frano, P. Popovich, V. Hinkov, P.Wochner,M.Castro-Colin,E. Detemple,V. K. Malik, C. Bernhard, T. Prokscha, A. Suter, Z. Salman, E. Morenzoni, G. Cristiani, H.-U. Habermeier, and B. Keimer, Dimensionality control of electronic phase transitions in nickel-oxide superlattices, Science 332, 937 (2011).

${ }^{30}$ See Supplementary Material: S1: Emergence of $\mathrm{Mn}^{2+}$ in LSMO films and influence on XLD and XMCD; S2: Area under XLD evaluated in different energy regions; S3: XMLD at normal incidence in LSMO films

${ }^{31}$ W.B. Wu, D.J. Huang, C.M. Huang, C.H. Hsu, C.F. Chang, H.J. Lin, and C.T. Chen, Orbital polarization and JahnTeller distortion of strained $\mathrm{La}_{0.5} \mathrm{Sr}_{0.5} \mathrm{MnO}_{3}$ thin films, J. Magn. Magn. Mater. 310, 813 (2007).

32 M. J. Calderon, L. Brey and F. Guinea, Surface electronic structure and magnetic properties of doped manganites, Phys. Rev. B 60, 6698 (1999).

33 D. Pesquera, G. Herranz, A. Barla, E. Pellegrin, F. Bondino, E. Magnano, F. Sánchez, and J. Fontcuberta, Surface symmetry-breaking and strain effects on orbital occupancy in transition metal perovskite epitaxial films, Nat. Commun. 3, 1189 (2012).

${ }^{34}$ A. Tebano, C. Aruta, S. Sanna, P. Medaglia, G. Balestrino, A. Sidorenko, R. De Renzi, G. Ghiringhelli, L. Braicovich, V. Bisogni, and N. Brookes, Evidence of orbital reconstruction at interfaces in ultrathin $\mathrm{La}_{0.67} \mathrm{Sr}_{0.33} \mathrm{MnO}_{3}$ films, Phys. Rev. Lett. 100, 137401 (2008).

35 J. Stöhr and H. König., Determination of spin- and orbital-moment anisotropies in transition metals by angledependent x-ray magnetic circular dichroism, Phys. Rev. Lett. 75, 3748 (1995)

${ }^{36}$ M. J. Han, C. A. Marianetti, and A. J. Millis, Chemical control of orbital polarization in artificially structured transition-metal oxides: $\mathrm{La}_{2} \mathrm{NiXO}_{6}(\mathrm{X}=\mathrm{B}, \mathrm{Al}, \mathrm{Ga}, \mathrm{In}$ ) from first principles, Phys. Rev. B 82, 134408 (2010).

${ }^{37}$ O.E. Peil, M. Ferrero, and A. Georges, Orbital polarization in strained $\mathrm{LaNiO}_{3}$ : Structural distortions and correlation effects, Phys. Rev. B 90, 045128 (2014).

${ }^{38}$ K. Kuepper, F. Bondino, K. C. Prince, M. Zangrando, M. Zacchigna, A. F. Takás, T. Crainic, M. Matteucci, F. Parmigiani, A Winiarski, V. R. Galakhov, Y. M. Mukovskii, and M. Neumann, Direct investigation of orbital ordering in a colossal magnetoresistance manganite by means of X-ray linear dichroism at the Mn L edge, J. Phys. Chem. B 109, 33, 15667 (2005). 
${ }^{39}$ H. Wu, C. F. Chang, O. Schumann, Z. Hu, J. C. Cezar, T. Burnus, N. Hollmann, N. B. Brookes, A. Tanaka, M. Braden, L. H. Tjeng, and D. I. Khomskii, Orbital order in $\mathrm{La}_{0.5} \mathrm{Sr}_{1.5} \mathrm{MnO}_{4}$ : Beyond a common local Jahn-Teller picture, Phys. Rev. B 84, 155126 (2011)

${ }^{40}$ A. O. Sboychakov, K. I. Kugel, A. L. Rakhmanov, and D. I. Khomskii, Relationship between orbital structure and lattice distortions in Jahn-Teller systems, Phys. Rev. B 83, 205123 (2011)

${ }^{41}$ C. Aruta, M. Minola, A. Galdi, R. Ciancio, A. Yu. Petrov, N. B. Brookes, G. Ghiringhelli, L. Maritato, and P. Orgiani, Off-stoichiometry effect on orbital order in A-site manganites probed by $\mathrm{x}$-ray absorption spectroscopy, Phys. Rev. B 86, 115132 (2012)

42 B. T. Thole, P. Carra, F. Sette, and G. van der Laan, X-ray circular dichroism as a probe of orbital magnetization, Phys. Rev. Lett. 68, 1943 (1992); P. Carra, B. T. Thole, M. Altarelli, and X. Wang, X-ray circular dichroism and local magnetic fields, Phys. Rev. Lett. 70, 694 (1993)

43 J. Stöhr, X-ray magnetic circular dichroism spectroscopy of transition metal thin films, J. of Elec. Spec. and Related Phenomena 75, 253 (1995).

44 C. Aruta, G. Ghiringhelli, V. Bisogni, L. Braicovich, N. Brookes, A. Tebano, and G. Balestrino, Orbital occupation, atomic moments and magnetic ordering at interfaces of manganite thin films, Phys. Rev. B 80, 014431, (2009).

${ }^{45}$ S. Valencia, L. Peña, Z. Konstantinovic, L. Balcells, R. Galceran, D. Schmitz, F. Sandiumenge, M. Casanove, and B. Martínez, Intrinsic antiferromagnetic/insulating phase at manganite surfaces and interfaces, J. Phys. Condens. Matter 26, 166001 (2014).

${ }^{46}$ Y. Takamura, F. Yang, N. Kemik, E. Arenholz, M. D. Biegalski, and H. M. Christen, Competing interactions in ferromagnetic/antiferromagnetic perovskite superlattices, Phys. Rev. B 80, 180417 (2009).

${ }^{47}$ D. Pesquera, M. Wojcik, E. Jedryka, V. Laukhin, N. Dix, F. Sánchez, and J. Fontcuberta, Interface and bulk charge localization in manganite thin films, Adv. Mater. Interfaces, 1: 1400079. doi: 10.1002/admi.201400079 (2014)

${ }^{48}$ A. A. Sidorenko, G. Allodi, R. De Renzi, G. Balestrino, and M. Angeloni, ${ }^{55} \mathrm{Mn}$ NMR and magnetization studies of $\mathrm{La}_{0.67} \mathrm{Sr}_{0.33} \mathrm{MnO}_{3}$ thin films, Phys. Rev. B 73, 054406 (2006)

${ }^{49}$ S. Brivio, C. Magen, A. A. Sidorenko, D. Petti, M. Cantoni, M. Finazzi, F. Ciccacci, R. De Renzi, M. Varela, S. Picozzi, and R. Bertacco, Effects of $A u$ nanoparticles on the magnetic and transport properties of $\mathrm{La}_{0.67} \mathrm{Sr}_{0.33} \mathrm{MnO}_{3}$ ultrathin layers, Phys. Rev. B 81, 094410 (2010).

${ }^{50}$ I.C. Infante, F. Sánchez, J. Fontcuberta, M. Wojcik, E. Jedryka, S. Estradé, F. Peiró, J. Arbiol, V. Laukhin, and J.P. Espinós, Elastic and orbital effects on thickness-dependent properties of manganite thin films, Phys. Rev. B 76, 224415 (2007).

${ }^{51}$ G Allodi, R De Renzi, K Zheng, S Sanna, A Sidorenko, C Baumann,L Righi, F Orlandi and G Calestani, Band filling effect on polaron localization in $\mathrm{La}_{1-\mathrm{x}}\left(\mathrm{Ca}_{\mathrm{y}} \mathrm{Sr}_{1-\mathrm{y}}\right){ }_{x} \mathrm{MnO}_{3}$ manganites, J. Phys.: Condens. Matter. 26, 266004 (2014).

${ }^{52}$ T. Kubo, A. Hirai, and H. Abe, Mn55 Nuclear Magnetic Resonance of the $\mathrm{Mn}^{3+}$ Ion Located at the B-Site in Manganese Ferrite Single Crystal-Anisotropic Hyperfine Field Due to the Local Jahn-Teller Distortion, J. Phys. Soc. Jpn. 26, 1094-1109 (1969).

${ }^{53}$ G. Allodi, M. Bimbi, and R. De Renzi, C. Baumann, M. Apostu, R. Suryanarayanan and A. Revcolevschi, Magnetic order in the double-layer manganites $\left(\mathrm{La}_{1-2} \mathrm{Pr}_{2}\right)_{1.2} \mathrm{Sr}_{1.8} \mathrm{Mn}_{2} \mathrm{O}_{7}$ : Intrinsic properties and role of intergrowth, Phys. Rev. B 78, 064420 (2008).

${ }^{54}$ D. Rybicki, Nuclear magnetic resonance study of selected Ruddlesden-Popper manganites, PhD Thesis, AGH University of Sciences and Technology (Krakow, Poland) and Charles University (Prague, Czech Republic), (2007) (winntbg.bg.agh.edu.pl/rozprawy/9786/full9786.pdf).

55 O. Chmaissem, B. Dabrowski, S. Kolesnik, J. Mais, J. D. Jorgensen, and S. Short, Structural and magnetic phase diagrams of $\mathrm{La}_{1-x} \mathrm{Sr}_{x} \mathrm{MnO}_{3}$ and $\mathrm{Pr}_{1-y} \mathrm{Sr}_{y} \mathrm{MnO}_{3}$, Phys. Rev. B 67, 094431 (2003). 